\title{
On the separation of component spectra in binary and higher-multiplicity stellar systems: bias progression and spurious patterns
}

\author{
H. Hensberge ${ }^{1}$, S. Ilijić ${ }^{2}$, and K. B. V. Torres ${ }^{1,3}$ \\ 1 Royal Observatory of Belgium, Ringlaan 3, 1180 Brussels, Belgium \\ e-mail: herman.hensberge@oma.be \\ 2 Department of Physics, Faculty of Electrical Engineering and Computing, University of Zagreb, Unska 3, 10000 Zagreb, Croatia \\ 3 Departamento de Fisica, Universidade Federal de Minas Gerais, CP 702, Belo Horizonte 30123-970 MG, Brazil
}

Received 9 November 2007 / Accepted 9 February 2008

\begin{abstract}
Context. Powerful methods are available to reconstruct the spectra of stars in orbit around each other, using a time-series of observed, composite spectra. They act either on the Fourier components of the observed spectra, or directly on these spectra in a velocity grid. Aims. We discuss under which conditions spurious patterns can appear in reconstructed spectra, either as a consequence of (quasi)degeneracy of the equations or as a consequence of bias in the observed spectra. Also we discuss the equivalence of Fourier and direct methods in practice.

Methods. We show under which conditions the equations degenerate, and how to evaluate this. We pay special attention to spectra of binary stars and triple systems analysed in Fourier space. We apply the theory to real data sets and to artificial data sets with several types of data-reduction bias constructed to illustrate degeneracies and the transfer of bias to the reconstructed spectra.

Results. Quasi-degeneracy of the equations depends on the lack of significant time-variability in the relative light contribution of the stars, on the length of the spectral interval in units of the involved Doppler shifts, on the presence of very faint stellar components, and on the distribution of the observations over the orbital phases. Eclipse spectra, possibly used with different weight in low- and highfrequency Fourier modes, remove quasi-degeneracies. But when the normalisation of the observed spectra is biased in a systematic way with relation to the orbital phase, then the bias amplifies strongly in the reconstructed spectra, particularly for the faintest components. Wavelength-locked bias is transferred more strongly to the spectrum of the star with the lowest velocity amplitude. Unrecognized variations in line strength lead to bias that is larger in spectral regions with high line-density. Most importantly, the bias in all reconstructed spectra is coupled in a unique, predictable way. This coupling allows us to design robust procedures for the removal of bias from the reconstructed spectra.

Conclusions. When the spectral features of all stars visible in the observed spectra undergo a significant time-variable dilution effect, then the reconstructed spectra are well-defined. Otherwise, spurious patterns, mostly of low frequency, may be superimposed on the reconstructed spectra. The analysis presented in this paper allows observers to optimise their observing strategy, gives insight in the origin of spurious patterns and indicates ways either to suppress such patterns or to remove them a posteriori from the reconstructed spectra. In this way, a broader range of astrophysical analyses can be applied to the component spectra.
\end{abstract}

Key words. stars: binaries: spectroscopic - methods: data analysis - techniques: spectroscopic - stars: binaries: eclipsing

\section{Introduction}

Binary stars are the principal source of stellar masses, by observation of the orbital Doppler shifts of the components. While conceptually simple, the precision of the measurements is hampered by the dilution of the observed spectral lines by the combined continuum of all components, and the accuracy is degraded by the confusing contribution of spectral lines of different components to the same observed spectral feature. Application of the cross-correlation technique has significantly reduced the disadvantages of position measurements on individual spectral features, in particular for rich, sharp-lined spectra. Efficient application to multiple-component spectra has been worked out by Zucker \& Mazeh (1994) and Zucker et al. (1995). The most serious drawbacks of the technique are the need for realistic template spectra and for separating the contributions of the components to the cross-correlation function (CCF). Spectra with intrinsically heavily-blended contributions are usually disregarded and, more generally, the dependence of the shape of the CCF on chance coincidences of different spectral lines displaces the multiple peaks of the $\mathrm{CCF}$, in a complex way, from the positions corresponding to the radial velocities of each component.

These limitations have motivated the development of techniques avoiding the direct measurement of Doppler shifts. The disentangling of multiple-component spectra aims at the selfconsistent solution for the relative intensities in the component spectra and the orbital parameters. This technique consists of an iterative search for the orbital parameters and the determination of the associated contribution of each component to all observed spectra. The technique has been developed by Simon \& Sturm (1994) in terms of the velocity-binned spectra, and independently by Hadrava (1995) in terms of the Fourier 
components of those spectra. Earlier, Bagnuolo \& Gies (1991) solved the simpler problem of separating the spectra of the different components for pre-determined radial-velocity shifts (which is part of the disentangling process) with a tomographic technique. Recently, González \& Levato (2006) promoted a tomography-like method with iterations on the Doppler shifts, alternately using an estimation of the spectrum of one component to predict the spectrum, as well as the Doppler shift, of the other one.

These techniques involve a very large number of parameters, of the order of the sum of the number of spectral bins in all intrinsic component spectra. The assumption that the best representation (in a least-square sense) of the observed spectra, in terms of all these parameters, delivers the true orbital parameters and true component spectra, has not yet been proven mathematically. Both practical experience and experiments with artificial data (Hynes \& Maxted 1998; Ilijić et al. 2001; González \& Levato 2006) support its validity for representative data sets, however, with the restriction of a limited capacity to reconstruct the low-frequency shape of the component spectra (scales much larger than the Doppler shifts) in the case of (quasi)-constant light ratios between components. This includes the comparison with spectra directly observed in total eclipse (Simon \& Sturm 1994). Practical applications encompass a large variety of stellar spectra and quality of input spectra. Lists of applications are found in Gies (2004); Holmgren (2004); and Hensberge \& Pavlovski (2007).

In spite of these efforts to validate the method, several interesting questions related to the stability of the solution process and its dependence on bias in the input data remain unanswered, such as:

- How do we evaluate the stability of the solution process in terms of the input data set?

- How do we plan observations to increase the stability of the solutions?

- How is bias in the input spectra transferred to the component spectra and the orbital parameters?

- How strong is the coupling between bias in the orbital parameters and in the component spectra?

- To what extent do bias progression and numerical instabilities depend on the details of the applied method?

In many papers, the use of spectrum reconstruction methods is restricted to the determination of orbital parameters and global characteristics of the component spectra, such as spectral types and rotation velocity. The appearance of low-frequency spurious patterns diminished the confidence in a detailed analysis of the reconstructed spectra, inclusive of the broad hydrogen lines, although the individual spectral lines are rich in information on the structure, temperature, gravity, and chemical composition of the stellar atmosphere. It is the aim of this paper to identify the origin of spurious patterns in the reconstructed spectra and to indicate how to reduce these significantly in a number of cases. This should motivate researchers to analyse the component spectra with confidence, and to identify better the situations in which caution is warranted.

In this paper, we study the process of the separation of the component spectra, with emphasis on binary stars and triple systems (although several aspects are straightforward to generalise to higher multiplicities). The discussion of aspects related to disentangling rather than separation of component spectra is not included here. In other words, effects related to the optimisation of the orbital parameters are presently neglected.
In Sect. 2, we recall the mathematical formulation of the basic problem. The numerical solution methods are summarised in Sect. 3, and it is shown under which conditions the Fourier approach and the direct approach are equivalent. Then, we concentrate on the Fourier approach and introduce condition numbers to study under which conditions the set of equations for a specific Fourier mode degenerates or becomes numerically unstable (Sect. 4). We develop, in detail, the case of a binary star and a triple system (Sect. 4.1) and discuss briefly to what extent the conclusions are also valid for the direct approach (Sect. 4.2). Thereafter, we compute the condition numbers for representative sets of observed spectra and discuss their usefulness in improving the reconstructed spectra (Sect. 4.3). In Sect. 5, we consider the progression of bias in the observed spectra to the reconstructed component spectra. Finally (Sect. 6), we discuss how to recognise and how to remove spurious patterns in a consistent way from the reconstructed spectra, and then conclude with the outline of a work procedure, from the preparation of the observations until the evaluation of the quality of the reconstructed spectra (Sect. 7).

\section{Mathematical formulation}

Consider the case that light from a multiple stellar system with $K$ components $k=1 . . K$ adds up (linearly) to an observed spectrum, i.e., one that does not resolve the contributing components spatially. Time-dependent relative Doppler shifts $v_{k j}$ at times $t_{j}$, $j=1, \ldots, J$ and, possibly, variations in the relative light ratios produce changes in the observed spectra at different epochs. Hence, representing the normalised component spectra as functions $x_{k}(\ln \lambda)$ of the logarithm of the wavelength, the normalised observed spectra $y_{j}(\ln \lambda)$ satisfy

$y_{j}(\ln \lambda)=\sum_{k=1}^{K} \ell_{k j} x_{k}\left(\ln \lambda-v_{k j} / c\right)+$ noise

where $\ell_{k j}$ is the light-factor $\left(\sum_{k=1}^{K} \ell_{k j}=1\right)$ and $v_{k j} / c$ is the orbital radial velocity, in units of the speed of light, of component $k$ at time $t_{j}$. This implies simple physics, disregarding any variations in line shape due to partial eclipses, ellipsoidality of the stars, variation of physical conditions over the stellar surface or variation of the light factors over the (short) wavelength range over which the component spectra are reconstructed. Strictly speaking, it also excludes components with a multiplicative nature such as interstellar absorption features, telluric lines and "chromospheric eclipses". A discussion on how to extend the method to deal with some of these more complex situations, can be found in Hadrava (2004a, 2006).

Note that in the case of constant light-ratios $\left(\ell_{k j}=\ell_{k}\right)$ the solution $\left\{\ell_{k}, x_{k}(\ln \lambda), v_{k j}\right\}$ is not unique, since

$$
\left\{\ell_{k}^{\prime}, 1-\frac{\ell_{k}}{\ell_{k}^{\prime}}\left(1-x_{k}(\ln \lambda)\right), v_{k j}\right\}
$$

then also reproduces the observed spectra equally well:

$$
\sum_{k=1}^{K} \ell_{k}^{\prime}\left(1-\frac{\ell_{k}}{\ell_{k}^{\prime}}\left(1-x_{k}\right)\right)=\sum_{k=1}^{K} \ell_{k}^{\prime}-\sum_{k=1}^{K} \ell_{k}+\sum_{k=1}^{K} \ell_{k} x_{k}=\sum_{k=1}^{K} \ell_{k} x_{k} .
$$

In this case, the shape (but not the strength) of all spectral features in the intrinsic component spectra is reconstructed correctly for any choice of $\ell_{k}$, but additional external information is required to transform these "quasi-spectra" to the true normalised spectra. In a system that includes components showing 
light-variability, the same non-uniqueness applies to any subset of components whose light does not change with time, or more generally, to a subset of components with mutual constant lightratios $r_{k k^{\prime}}=\ell_{k j} / \ell_{k^{\prime} j}$.

Only a subset of these mathematical solutions are acceptable, since a spectrum should have non-negative intensity at all wavelengths. In this respect, the choice of wavelength intervals containing very deep absorption lines resolves much of the indeterminacy in the case of constant light-ratios (see e.g. Griffin 2002; Frémat et al. 2005). Several additional physics constraints may help in particular cases (e.g., knowledge about continuum windows, or, less direct, the requirement to obtain consistent abundances from weak and strong lines of the same ion). Photometry may give useful clues to the time variability of light ratios, although not necessarily in an exact quantitative way in view of the different responses of the photometric passbands and the spectrograph. In principle, information on the light ratios can be derived from the reconstruction of the spectra, using the $\ell_{k j}$ as variables (Hadrava 1997). This has led to the spectroscopic detection of eclipses (e.g., Holmgren et al. 1997; González \& Levato 2006). In the case of more subtle light variability, the precision obtained in photometric investigations may largely exceed the precision obtainable in a spectrum analysis. The key point is that any misplacement of the continuum translates directly to an apparent change of the line depths in the spectrum. Such misplacement may be related to the variable blending of spectral lines of Doppler-shifted components, changing the continuum windows, and to different signal-to-noise ratios in the spectra. The effects of continuum misplacements are larger for components with shallower spectral features in the observed spectra. In Sect. 5, we further discuss bias in the normalised spectra.

In practice, the spectra observed at times $t_{j}, j=1 \ldots J$ are discretised on a grid of log-wavelength points $\ln \lambda_{j i}, i=1 \ldots I_{j}$, which may differ for each observation (but covering roughly the same wavelength interval) and the normalised intensities $y_{j i}$ have uncertainties $\sigma_{j i}$.

\section{Numerical solution}

In order to reconstruct the component spectra $x_{k}(\ln \lambda)$, Eq. (1) must be expressed as an algebraic problem. In this respect, the component spectra $x_{k}(\ln \lambda)$ are written as a linear interpolation of a discrete set of normalised intensities $x_{k n}, n=1 \ldots N_{k}$, corresponding to a grid of $\log$-wavelength points $\ln \lambda_{k n}$. Each component may be sampled on a different grid and no strict regularity in sampling in log-wavelength is required. The natural resolution on the detector can be used without applying rebinning. For instance, log-wavelength bins may have shifts of a fraction of a bin in different spectra, may have a (slightly) different size in different spectra or spectral orders, etc. However, the features in the spectra should be sampled sufficiently well and similar such that the applied interpolation will not bias the data significantly. The grids must also cover, for each component, a wavelength range that is wide enough to include the extreme values of logwavelength appearing in the argument of $x_{k}$ in (1). Then,

$x_{k}\left(\ln \lambda_{j i}-\frac{v_{k j}}{c}\right)=\left(1-f_{j i k}\right) x_{k n_{j i k}}+f_{j i k} x_{k\left(n_{j i k}+1\right)}$,

where $n_{j i k}$ satisfies $\ln \lambda_{k n_{j i k}} \leq \ln \lambda_{j i}-\frac{v_{k j}}{c} \leq \ln \lambda_{k\left(n_{j i k}+1\right)}$, and

$f_{j i k}=\frac{\ln \lambda_{j i}-\frac{v_{k j}}{c}-\ln \lambda_{k n_{j i k}}}{\ln \lambda_{k\left(n_{j i k}+1\right)}-\ln \lambda_{k n_{j i k}}}$ is the linear interpolation coefficient. Substituting (2) into (1), taking into account (3), gives a system of $\sum_{j=1}^{J} I_{j}$ linear equations in $\sum_{k=1}^{K} N_{k}$ unknowns $\left(x_{k n}\right)$,

$\frac{y_{j i}}{\sigma_{j i}}=\frac{1}{\sigma_{j i}} \sum_{k=1}^{K} \ell_{k j}\left[\left(1-f_{j i k}\right) x_{k n_{j i k}}+f_{j i k} x_{k\left(n_{j i k}+1\right)}\right]$,

the noise/residual term being omitted.

We assume that the system is overdetermined (i.e., $\left.\sum_{j=1}^{J} I_{j}>\sum_{k=1}^{K} N_{k}\right)$, and seek the least-squares solution. Note that each equation in the system (4) has been divided by the uncertainty $\sigma_{j i}$ to ensure proper weighting of each data point in the sum of squared residuals. The weighting scheme may include masking of detector blemishes or of data altered by physical processes not included in the model. Assigning weight zero to wavelength bins affected by telluric or interstellar features is the correct way to eliminate the effect of components with a multiplicative, and not an additive, impact on the observed spectra. As Hadrava (2006) shows, such components can be treated in certain cases in good approximation as an additional additive component during the separation process. This is only the case when the telluric or interstellar lines are superposed on similar levels of normalised intensity in all spectra. But a more rigid method is mandatory, for example, in the case of an F-type star which has an interstellar component superposed on the very strong $\mathrm{Na}$ I D lines, while the latter move over a Doppler range of the order of the width of these lines or larger.

Concatenating the values $y_{j i} / \sigma_{j i}$ on the lefthand side into a vector of length $\sum_{j} I_{j}$, which we denote $\boldsymbol{b}$, and the (unknown) relative intensities of the component spectra $x_{k n}$ into the vector $\boldsymbol{x}$ of length $\sum_{k} N_{k}$, the system of equations can be written as $\boldsymbol{A} \cdot \boldsymbol{x}=$ $\boldsymbol{b}$ where $\boldsymbol{A}$ is the $\sum_{j} I_{j}$ by $\sum_{k} N_{k}$ matrix further on referred to as the design matrix. Alternatively, one could consider obtaining the least-squares solution to (4) by solving the system $\boldsymbol{A}^{\mathrm{T}} \boldsymbol{A} \cdot \boldsymbol{x}=$ $\boldsymbol{A}^{\mathrm{T}} \cdot \boldsymbol{b}$, where the square matrix $\boldsymbol{A}^{\mathrm{T}} \boldsymbol{A}$ is further on referred to as the normal matrix.

In order to handle cases in which the design matrix or the normal matrix is ill-conditioned or singular, the least-square solution, i.e., the vector $\boldsymbol{x}$ that minimizes $\chi^{2}=|\boldsymbol{b}-\boldsymbol{A} \cdot \boldsymbol{x}|^{2}$ or $\chi^{2}=\left|\boldsymbol{A}^{\mathrm{T}} \cdot \boldsymbol{b}-\boldsymbol{A}^{\mathrm{T}} \boldsymbol{A} \cdot \boldsymbol{x}\right|^{2}$, is obtained preferentially by the singular value decomposition (SVD) of the design or the normal matrix, and construction of the pseudo-inverse matrix (Press et al. 1992). While the solution based on the normal matrix is computationally less-demanding, the application of SVD to the design matrix is less susceptible to numerical instabilities. These issues are further discussed in Sect. 4.

A computationally more efficient separation algorithm has been formulated in terms of the discrete Fourier transforms (DFT) of the spectra (Hadrava 1995), however, at the expense of the following limitations. All $J$ observed spectra must be sampled on a common grid of $N$ points with constant step in logwavelength, $\Delta \ln \lambda$, related to the radial velocity resolution of the grid, $v_{\mathrm{g}}$, by $\Delta \ln \lambda=\Delta \lambda / \lambda=v_{\mathrm{g}} / c$. The same grid must be used for the $K$ component spectra and the spectra are considered to be periodic functions of log-wavelength, the period being the full length of the grid. Furthermore, all data points within one observed spectrum are assigned equal uncertainty $\sigma_{j}$, and therefore equal fitting weight. In Sect. 4.1.1, we will argue for different weighting schemes in low- and high-frequency Fourier modes $m$, i.e., $\sigma_{j, m}$. The DFT of the spectra are given by

$\tilde{y}_{j, m}=\sum_{n=1}^{N} y_{j n} \mathrm{e}^{-2 \pi \mathrm{i} m n / N} \quad$ and $\quad \tilde{x}_{k, m}=\sum_{n=1}^{N} x_{k n} \mathrm{e}^{-2 \pi \mathrm{i} m n / N}$. 
Since the spectra $x_{k n}$ and $y_{j n}$ are real, the DFT amplitudes $\tilde{x}_{k, m}$ and $\tilde{y}_{j, m}$ at modes $\pm m$ are complex-conjugates of one another, and it suffices to solve for the modes $m=0 \ldots N / 2$ for $N$ even or $m=0 \ldots(N-1) / 2$ for $N$ odd. According to the convolution theorem, the shift of $x_{k n}$ by $\beta$ pixels in the log-wavelength grid corresponds to multiplying its DFT by the factor $\mathrm{e}^{-2 \pi \mathrm{i} m \beta / N}$. This allows one to write the analogue of Eq. (4) as

$$
\begin{aligned}
\frac{\tilde{y}_{j, m}}{\sigma_{j, m}}= & \frac{1}{\sigma_{j, m}} \sum_{k=1}^{K} \ell_{k j}\left[\left(\beta_{k j}^{+}-\beta_{k j}\right) \mathrm{e}^{-2 \pi \mathrm{i} m \beta_{k j}^{-} / N}\right. \\
& \left.+\left(\beta_{k j}-\beta_{k j}^{-}\right) \mathrm{e}^{-2 \pi \mathrm{i} m \beta_{k j}^{+} / N}\right] \tilde{x}_{k, m}
\end{aligned}
$$

where $\beta_{k j}=v_{k j} / v_{\text {grid }}$ is the radial velocity of component $k$ at time $t_{j}$ in units of the radial velocity resolution of the logwavelength grid (i.e. the shift expressed in "pixel units"), while $\beta_{k j}^{ \pm}$are the nearest integers from above and from below. The original set of $J \times N$ equations in $K \times N$ unknowns is now uncoupled into $N / 2+1$ (for $N$ even) or $(N+1) / 2$ (for $N$ odd) sets of $J$ equations in $K$ unknowns, each small set of equations with few unknowns corresponding to one Fourier mode $m$. Note that the system of equations corresponding to $m=0$, and to $m=N / 2$ for $N$ even is real, while all others are complex.

For each Fourier mode the system of equations can be written as $\boldsymbol{F}_{m} \cdot \tilde{\boldsymbol{x}}_{m}=\tilde{\boldsymbol{b}}_{m}$, where $\left(\tilde{\boldsymbol{x}}_{m}\right)_{k}=\tilde{x}_{k, m},\left(\tilde{\boldsymbol{b}}_{m}\right)_{j}=\tilde{y}_{j, m} / \sigma_{j, m}$, and $\boldsymbol{F}_{m}$ is a $J$ by $K$ matrix. Contrary to the direct formulation in terms of the spectra in the log-wavelength grid, ill-conditioned Fourier modes (e.g., low-frequency modes, see also Sect. 4.1.2) are easily identified and may be masked during the optimalisation of parameters or at least in early stages of the optimalisation process. For $J>K$, the systems are overdetermined and the least-squares solution must be obtained for each $m$, separately. According to the power theorem, the least squares solution in the Fourier domain corresponds to the least-squares solution of the original system of equations. Since the design matrix $\boldsymbol{F}_{m}$ may be singular or ill-conditioned, the SVD of $\boldsymbol{F}_{m}$ should be performed.

\subsection{Equivalence of methods}

The result obtained with Fourier transforms can be reproduced exactly in velocity space with an appropriate re-definition of the design matrix. However, straightforward application of both methods as implemented in available numerical codes leads, in general, to slightly different results. This is due to the fact that the Fourier transforms imply that the spectra are periodic functions and thus connect the information at both edges and to different technical options in the codes, related to data resampling and to the solution of ill-conditioned equations.

The basic difference in the solution procedures is schematised in Fig. 1, showing the design matrix for the classical velocity-space algorithm and for an algorithm that reproduces exactly the results obtained in Fourier space - given all technical details are identical - but operates in velocity space. While the classical algorithm uses a design matrix extending, for each component, from the bluest log wavelength to the reddest one in the reconstructed component spectrum, and hence exceeds the interval covered in any of the observed spectra, the design matrix in the Fourier-equivalent algorithm encompasses only the observed log-wavelength interval. The information from inside this range that is Doppler-shifted out of the interval at some orbital phases is wrapped around to the other side of the logwavelength interval. Using various artificial and observed data sets, we tested that the velocity space algorithm with the Fourierequivalent design matrix is equivalent to the Fourier-domain
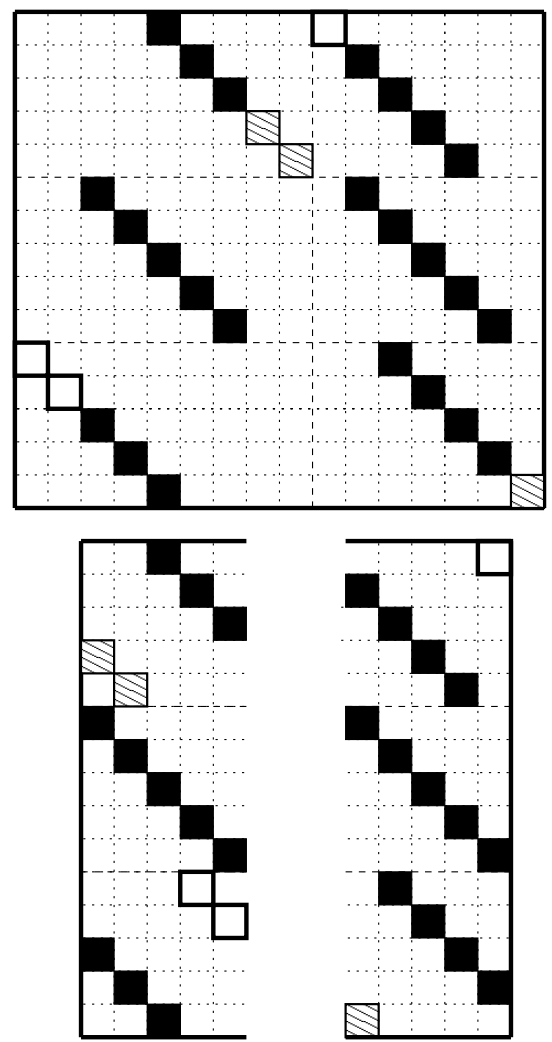

Fig. 1. Design matrix for the classical velocity-space algorithm (upper panel) and the Fourier-equivalent algorithm (lower panel) for a case of two components and three observed spectra. Only elements represented by filled, shaded or thick-lined squares differ from 0 . The submatrices at the 9 respectively 5 lefthand columns refer to one component, those at the 7 respectively 5 righthand columns refer to the other component of a binary. The spectra corresponding to the 5-rows upper sub-matrices are shifted one pixel to the blue (right-hand side component), respectively, two pixel to the red, those in the middle 5 rows are unshifted, and those in the lower 5 rows have shifts with the sign opposed to those in the upper 5 rows. Shaded and thick-lined squares indicate non-zero elements wrapped around in the Fourier-equivalent design matrix due to red-shifts and blue-shifts, respectively, but not in the classical design matrix. Note the smaller size of the Fourier-equivalent matrix (exactly the selected observed interval) owing to the wrap-around of data. The Fourier-equivalent matrix is presented with an artificial "gap" between the central rows to align the corresponding columns in the two matrices.

solution when they both deal with the same technical assumptions. Analytical computations on few-pixel data strings are presented in Appendix A and in the Ph.D. thesis of K. B. V. Torres.

Having established the basic equivalence of the solution methods, in the next subsections attention is paid to the practical issues of the choice of the edges of the data strings and the definition of the sampling of input and output data. The impact of the choice of the type of solution algorithm for ill-conditioned sets of equations is delayed to Sect. 4.2, after a detailed discussion of numerical instabilities.

\subsubsection{Edge effects}

The reconstruction of the component spectra is badly constrained near the edges of the wavelength interval under consideration unless there is sufficient pure continuum at both edges, a situation which is not easily encountered in high-density line spectra or in spectra of fast-rotating stars. If the observed spectrum contains a faint component, it is even hard to estimate 


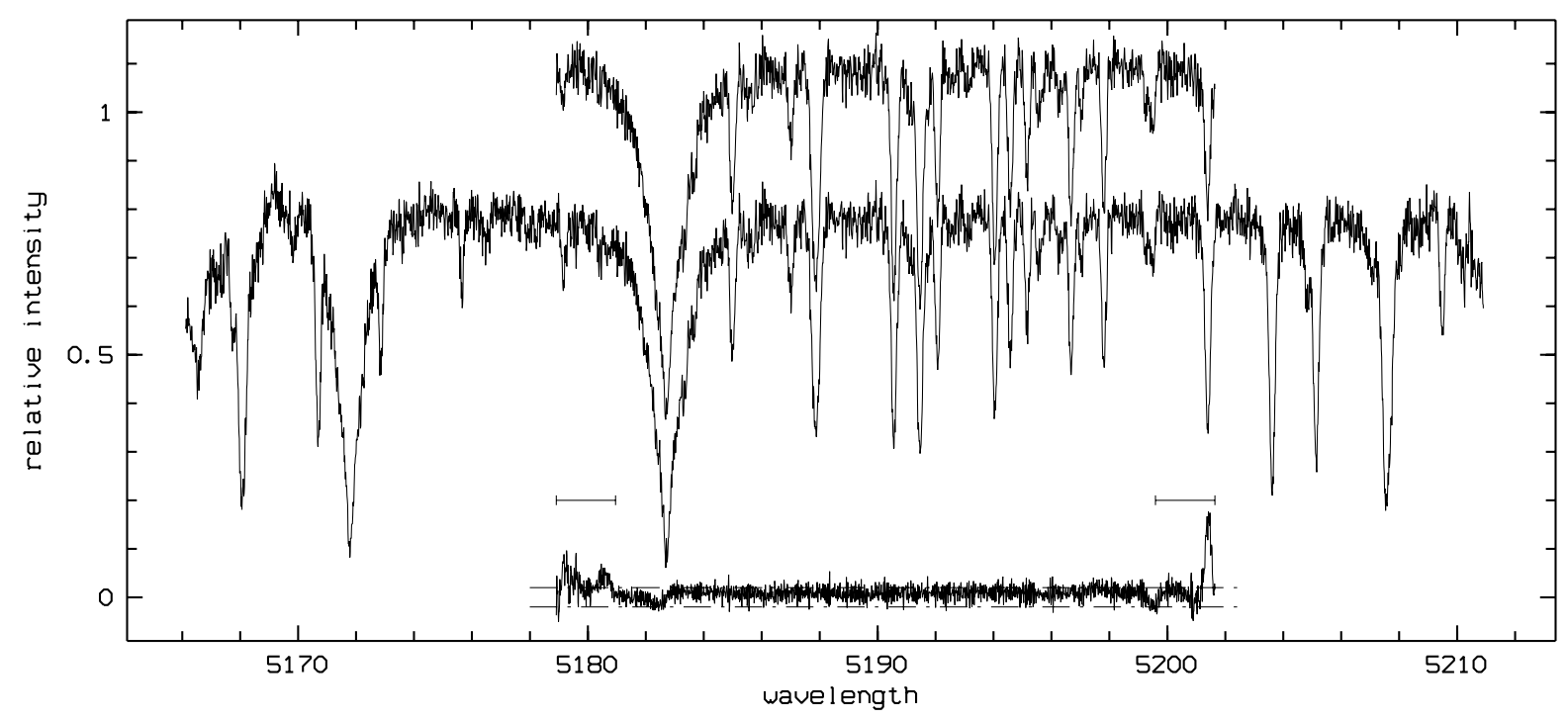

Fig. 2. Disentangled spectrum of one component of a late-type binary using 37 low signal-to-noise spectra and a shorter respectively longer wavelength region (2048 and 4096 bins) centred around $5190 \AA$ A. For clarity, vertical shifts are applied to the component spectra. The difference between the results is shown together with dot-dashed lines at the 2 percent level. Notice the deviations at the edges, especially at the long wavelength edge where a strong line is present. The size of the sum of the velocity amplitudes, $K_{1}+K_{2}$, is shown with horizontal bars near the edges of the smaller wavelength interval.

whether this component has strong spectral lines near the edges of the interval. The choice of the edges of the input spectra is potentially very important. With spectral lines crossing the edges during the orbital cycle, some of the observed spectra have a normalised intensity at the blue edge which differs from the red edge. Their Fourier representation shows then the Gibbsphenomenon, a wavy pattern of deviations from the true functions that travel inside the considered interval with quickly decreasing amplitude. Unfortunately, the choice of the edges is constrained most by implementations of the algorithm that uses the fast Fourier transform, which requires data strings with a number of bins equal to a power of 2 . In principle, the data can be resampled to bin sizes ensuring continuum near the edges and the required number of bins, but this sampling may violate other gridding requirements (see also Sect. 3.1.2).

It is not possible to follow the propagation of the Gibbs phenomenon through the solution process with mathematical rigidity. Experiments suggest that the characteristic fast decay of deviations toward the centre is conserved. In practice, a check on the Fourier representation of the observed spectra might be instructive for applications requiring the astrophysical interpretation of line strengths near the edges. The observable defect is that lines reconstructed near the edge of the interval (distance to the edge not significantly larger than the sum of the velocity amplitudes of the orbits) are badly reconstructed. An example is shown in Fig. 2. The influence of the unphysical treatment of the edges spreads only marginally over the whole interval: structure in the deviations between the reconstruction of the spectrum over a shorter and a longer wavelength range is only seen near the edges of the shorter interval, within twice the sum of the velocity amplitudes of the reconstructed components. In the remaining, more central part of the short interval the rms scatter amounts to 0.012 (with an offset in level between the two reconstructions of 0.008) and no structure was detected. The rms scatter expected in a straightforward weighted addition of the observed spectra is 0.008 and taking into account that the component shown contributes $64 \%$ of the observed light, the reconstructed spectrum is expected to have a rms noise of 0.012 .
In velocity space, only part of the observed spectra contribute to the reconstruction of the spectra around the edges of the observed log-wavelength interval. The equations, linking the reconstructed flux in bins near the edges to the flux in bins that are never Doppler-shifted inside the observed log-wavelength interval are missing. No "false" information is wrapped around, but the set of equations is still not well-closed.

Estimates on the presence of edge effects in practice can be obtained most easily by applying both direct and Fourier techniques, and by applying the reconstruction algorithm to overlapping spectral regions (i.e., with different edges). In conclusion, in the case that some observed spectra have lines near an edge of the selected wavelength interval, or that components are reconstructed that contribute only weakly to the observed spectra, we recommend that astrophysical analyses that depend on line strengths be limited to the part of the interval for which the whole spectral line is not closer to the edge than one or two times the sum of the largest velocity amplitudes.

\subsubsection{Grid definition}

Several aspects intervene when defining the sampling of the observed spectra and the output component spectra. On one hand, the natural sampling from the observational viewpoint is defined by the size of the pixels of the detector. With many echelle spectrographs, the size of the pixels is fairly constant over a large wavelength range when expressed in velocity units, but not when expressed in wavelength units. Hence, we discourage the common practice of resampling spectra in wavelength units, and thereafter, for application of the reconstruction method, of resampling those spectra to constant velocity steps. This leads to unnecessary resampling errors and increases the correlation between errors on consecutive pixels. Inasfar as later error estimations and least-squares algorithms are based on the assumption of independent errors on each spectral bin, they will turn out to be too optimistic.

On the other hand, the sampling of the input spectra fixes the resolution in the orbital velocity grid, assuming that no 
further resampling to another grid is done in the spectra separation algorithms. In the case that the bin size is not sufficiently small with respect to the orbital velocity, then a finer grid (with, admittedly, oversampled spectra) may be advantageous. The option to introduce input spectra with different sampling and require component spectra with another sampling is straightforward in velocity space. This option is useful for fast-rotating components in a wide system with low-velocity amplitudes.

\section{Indeterminacies and numerical instabilities}

The DFT formulation, with its independent, small sets of equations, offers the easiest possibility to investigate in depth under which conditions the separation of spectra may lead to singular or ill-conditioned solutions, even using observed spectra with infinite signal-to-noise ratio. The SVD of the design matrix $\boldsymbol{F}_{m}$ with $J$ rows and $K<J$ columns is obtained by decomposing it to a product of three matrices, $\boldsymbol{F}_{m}=\boldsymbol{U}_{m} \cdot \boldsymbol{W}_{m} \cdot \boldsymbol{V}_{m}^{\dagger}$ where $\boldsymbol{U}_{m}$ is a $J \times K$ and $\boldsymbol{V}_{m}$ a $K \times K$ unitary matrix $\left(\boldsymbol{U}_{m}^{\dagger} \boldsymbol{U}_{m}=\boldsymbol{V}_{m}^{\dagger} \boldsymbol{V}_{m}=\mathbf{1}\right)$, and $\boldsymbol{W}_{m}$ is a $K \times K$ diagonal matrix with elements $\omega_{m, k}$. The solution of the set of equations $\boldsymbol{F}_{m} \cdot \tilde{\boldsymbol{x}}_{m}=\tilde{\boldsymbol{b}}_{m}$ is then $\tilde{\boldsymbol{x}}_{m}=$ $\boldsymbol{V}_{m} \cdot\left[\operatorname{diag}\left(\omega_{m, k}^{-1}\right)\right] \cdot\left(\boldsymbol{U}_{m}^{\dagger} \cdot \tilde{\boldsymbol{b}}_{m}\right)$ where $\omega_{m, k}^{-1}$ is replaced by zero if $\omega_{m, k}=0$. The latter substitution singles out the solution vector with the smallest length. In practice, applying this substitution when $\omega_{m, k}$ is very small but non-zero gives the SVD solution for an ill-conditioned set of equations. This solution is known to be often better, in the sense of the residual $\left|\boldsymbol{F}_{m} \cdot \tilde{\boldsymbol{x}}_{m}-\tilde{\boldsymbol{b}}_{m}\right|$ being smaller, than the formal one provided by direct methods such as Gaussian elimination or $L U$ (lower and upper triangular matrix) decomposition.

The ratio $C_{m}$ of the largest to the smallest singular value of $\boldsymbol{F}_{m}, \omega_{m,+} / \omega_{m,-}$, is a worst-case estimate of loss of precision in solving a linear system defined by the design matrix. $C_{m}$ is called the condition number. It is infinite when the system is singular and orders of magnitude larger than unity when the system is illconditioned. The quantity $\log C$ gives an estimate of how many digits are lost in the solution. Ill-conditioned means roughly that $\log C$ is comparable to or larger than the precision of the matrix entries.

The singular values of the square normal matrix $\boldsymbol{F}_{m}^{\dagger} \boldsymbol{F}_{m}$, which are also its eigenvalues, equal the squares of the singular values of the non-square design matrix $\boldsymbol{F}_{m}$, since

$\boldsymbol{F}_{m}^{\dagger} \boldsymbol{F}_{m}=\boldsymbol{V}_{m} \cdot \boldsymbol{W}_{m}^{\dagger} \cdot \boldsymbol{U}_{m}^{\dagger} \cdot \boldsymbol{U}_{m} \cdot \boldsymbol{W}_{m} \cdot \boldsymbol{V}_{m}^{\dagger}=\boldsymbol{V}_{m} \cdot \boldsymbol{W}_{m}^{2} \cdot \boldsymbol{V}_{m}^{\dagger}$.

Therefore, the condition number associated with the normal matrix $\boldsymbol{F}_{m}^{\dagger} \boldsymbol{F}_{m}$ is the square of the condition number associated with the design matrix $\boldsymbol{F}_{m}$. The larger condition number for the normal matrix quantifies the increased sensitivity to numerical instabilities. SVD is best applied to the design matrix.

To obtain the eigenvalues of the normal matrix, it suffices to solve its characteristic equation, $\operatorname{det}\left(\boldsymbol{F}_{m}^{\dagger} \boldsymbol{F}_{m}-\omega^{2} \boldsymbol{I}\right)=0$, which may be written as

$$
\begin{aligned}
& \left(\omega^{2}\right)^{K}-\operatorname{Tr}\left(\boldsymbol{F}_{m}^{\dagger} \boldsymbol{F}_{m}\right)\left(\omega^{2}\right)^{K-1}+\operatorname{Tr}_{2}\left(\boldsymbol{F}_{m}^{\dagger} \boldsymbol{F}_{m}\right)\left(\omega^{2}\right)^{K-2}+\cdots \\
& +(-1)^{K-1} \operatorname{Tr}_{K-1}\left(\boldsymbol{F}_{m}^{\dagger} \boldsymbol{F}_{m}\right) \omega^{2}+(-1)^{K} \operatorname{det}\left(\boldsymbol{F}_{m}^{\dagger} \boldsymbol{F}_{m}\right)=0 .
\end{aligned}
$$

We follow common practice to suppress the index 1 for the trace of the first order, which equals the sum of the diagonal elements of the matrix.

A general lemma on determinants of a product of two rectangular matrices $\boldsymbol{G}(K \times J)$ and $\boldsymbol{H}(J \times K)$ with $K<J$ facilitates the computation of $\operatorname{det}\left(\boldsymbol{F}_{m}^{\dagger} \boldsymbol{F}_{m}\right)$. It states that (Thrall \& Tornheim 1963)

$$
\operatorname{det}(\boldsymbol{G H})=\sum_{\left(j_{1}<j_{2}<\ldots<j_{K}\right) \epsilon(1, \ldots, J)} \operatorname{det}\left(\boldsymbol{G}^{\prime}{ }_{j_{1}, \ldots, j_{K}}\right) \operatorname{det}\left(\boldsymbol{H}^{\prime}{ }_{j_{1}, \ldots, j_{K}}\right),
$$

where the summation selects all choices of $K$ different indices out of $J$. The matrix $\boldsymbol{G}^{\prime}{ }_{j_{1}, \ldots, j_{K}}$ is the square matrix of degree $K$ extracted from $\boldsymbol{G}$, keeping the columns $j_{1}, \ldots, j_{K}$, and $\boldsymbol{H}^{\prime}{ }_{j_{1}, \ldots, j_{K}}$ is the square matrix of degree $K$ extracted from $\boldsymbol{H}$, keeping the corresponding rows $j_{1}, \ldots, j_{K}$. In our case, each determinant is a sum of terms of the type

$(-1)^{s} \prod_{u=1}^{K} \frac{\ell_{k_{u} j_{u}}}{\sigma_{j_{u}, m}} \mathrm{e}^{ \pm 2 \pi \mathrm{i} \frac{m}{N} \beta_{k_{u} j_{u}}}$

with the sign in the exponential depending on whether $\boldsymbol{F}_{m}^{\dagger \prime}$ or $\boldsymbol{F}_{m}^{\prime}$ is considered, and $s$ is the number of interchanges needed to restore the order of the $K$ different indices $\left(k_{1}, k_{2}, \ldots, k_{K}\right)$ to their natural order $(1,2, \ldots, K)$. Here the velocity shifts are assumed to be integer-bin shifts to improve the readability of the expressions. The product of the determinants is a sum of terms of the type

$(-1)^{s+s^{\prime}} \prod_{u=1}^{K} \frac{\ell_{k_{u} j_{u}} \ell_{k_{u}^{\prime} j_{u}}}{\sigma_{j_{u}, m}^{2}} \mathrm{e}^{2 \pi \mathrm{i} \frac{m}{N}\left(\beta_{k_{u} j_{u}}-\beta_{k_{u}^{\prime} j_{u}}\right)}$

For some values of $u, k_{u}=k_{u}^{\prime}$, while for other values $k_{u} \neq k_{u}^{\prime}$. Therefore, this expression may be rewritten as

$$
\begin{aligned}
& (-1)^{s+s^{\prime}}\left[\prod_{u}^{k_{u}=k_{u}^{\prime}} \frac{\ell_{k_{u} j_{u}}^{2}}{\sigma_{j_{u}, m}^{2}}\right]\left[\prod_{u}^{k_{u} \neq k_{u}^{\prime}} \frac{\ell_{k_{u} j_{u}} \ell_{k_{u}^{\prime} j_{u}}}{\sigma_{j_{u}, m}^{2}}\right] \\
& \times \exp \left[2 \pi \mathrm{i} \frac{m}{N} \sum_{u}^{k_{u} \neq k_{u}^{\prime}}\left(\beta_{k_{u} j_{u}}-\beta_{k_{u}^{\prime} j_{u}}\right)\right]
\end{aligned}
$$

assuming a value of 1 for a product that does not contain any term. Finally, combining the terms in the sum which are a complex conjugate of each other, one finds that $\operatorname{det}\left(\boldsymbol{F}_{m}^{\dagger} \boldsymbol{F}_{m}\right)$ is a sum of terms of the type

$$
\begin{aligned}
& (-1)^{s+s^{\prime}}\left[\prod_{u}^{k_{u}=k_{u}^{\prime}} \frac{\ell_{k_{u} j_{u}}^{2}}{\sigma_{j_{u}, m}^{2}}\right]\left[\prod_{u}^{k_{u} \neq k_{u}^{\prime}} \frac{\ell_{k_{u} j_{u}} \ell_{k_{u}^{\prime} j_{u}}}{\sigma_{j_{u}, m}^{2}}\right] \\
& \times 2 \cos 2 \pi \frac{m}{N} \sum_{u}^{k_{u} \neq k_{u}^{\prime}}\left(\beta_{k_{u} j_{u}}-\beta_{k_{u}^{\prime} j_{u}}\right) .
\end{aligned}
$$

In the next section, explicit expressions are written down for systems with small multiplicity. It will turn out useful to define

$$
\begin{aligned}
L_{\text {indices }} & =\prod_{u}^{k_{u} \neq k_{u}^{\prime}} \frac{\ell_{k_{u} j_{u}} \ell_{k_{u}^{\prime} j_{u}}}{\sigma_{j_{u}, m}^{2}} \\
\delta_{\text {indices }} & =\sum_{u}^{k_{u} \neq k_{u}^{\prime}}\left(\beta_{k_{u} j_{u}}-\beta_{k_{u}^{\prime} j_{u}}\right)
\end{aligned}
$$

where the "indices" of $L$ and $\delta$ are the $k_{u}$ that belong to the set of values of $u$ with $k_{u} \neq k_{u}^{\prime}$, in ascending order, followed by the corresponding set of $j_{u}$. 


\subsection{The Fourier case: binary stars and triple systems}

An analytical solution for the condition number of the design matrix can be obtained for systems with low multiplicity. With $K=2$, the characteristic Eq. (6) reduces to

$\omega^{4}-\omega^{2} \sum_{j=1}^{J} \frac{\ell_{1 j}^{2}+\ell_{2 j}^{2}}{\sigma_{j, m}^{2}}+\operatorname{det}\left(\boldsymbol{F}_{m}^{\dagger} \boldsymbol{F}_{m}\right)=0$

where, using Eq. (8), the determinant is written as a sum of nonnegative terms:

$\operatorname{det}\left(\boldsymbol{F}_{m}^{\dagger} \boldsymbol{F}_{m}\right)=\sum_{j_{2}=2}^{J} \sum_{j_{1}=1}^{j_{2}-1} \frac{\Delta \ell_{12 j_{1} j_{2}}^{2}+2 L_{12 j_{1} j_{2}}\left(1-\cos 2 \pi \frac{m}{N} \delta_{12 j_{1} j_{2}}\right)}{\sigma_{j_{1}, m}^{2} \sigma_{j_{2}, m}^{2}}(10)$

with

$$
\begin{aligned}
\Delta \ell_{12 j_{1} j_{2}} & =\ell_{1 j_{1}} \ell_{2 j_{2}}-\ell_{1 j_{2}} \ell_{2 j_{1}} \\
& =\ell_{1 j_{1}}-\ell_{1 j_{2}}, \quad \text { since } \ell_{1 j}+\ell_{2 j}=1 ; \\
L_{12 j_{1} j_{2}} & =\ell_{1 j_{1}} \ell_{1 j_{2}} \ell_{2 j_{1}} \ell_{2 j_{2}} \\
\delta_{12 j_{1} j_{2}} & =\left(\beta_{2 j_{2}}-\beta_{1 j_{2}}\right)-\left(\beta_{2 j_{1}}-\beta_{1 j_{1}}\right) \\
& =\left(\beta_{1 j_{1}}-\beta_{1 j_{2}}\right)-\left(\beta_{2 j_{1}}-\beta_{2 j_{2}}\right) .
\end{aligned}
$$

The condition number $C_{m}$ associated with the design matrix $\boldsymbol{F}_{m}$ is

$C_{m}=\frac{\omega_{m,+}}{\omega_{m,-}}=\frac{\omega_{m,+}^{2}}{\operatorname{det}^{1 / 2}\left(\boldsymbol{F}_{m}^{\dagger} \boldsymbol{F}_{m}\right)}$

where $\omega_{m,+}$ and $\omega_{m,-}$ are the larger, respectively smaller root of Eq. (9). This leads directly to

$C_{m}=z_{m}+\sqrt{z_{m}^{2}-1}$ with

$z_{m}=\frac{\sum_{j=1}^{J} \frac{\ell_{1 j}^{2}+\ell_{2 j}^{2}}{\sigma_{j, m}^{2}}}{2 \operatorname{det}^{1 / 2}\left(\boldsymbol{F}_{m}^{\dagger} \boldsymbol{F}_{m}\right)}=\frac{\sum_{j=1}^{J} \frac{1+\left(\ell_{1 j}-\ell_{2 j}\right)^{2}}{\sigma_{j, m}^{2}}}{4 \operatorname{det}^{1 / 2}\left(\boldsymbol{F}_{m}^{\dagger} \boldsymbol{F}_{m}\right)}$.

For $K=3$, the characteristic equation is

$\omega^{6}-\omega^{4} \sum_{j=1}^{J} \frac{\ell_{1 j}^{2}+\ell_{2 j}^{2}+\ell_{3 j}^{2}}{\sigma_{j, m}^{2}}+\omega^{2} \operatorname{Tr}_{2}\left(\boldsymbol{F}_{m}^{\dagger} \boldsymbol{F}_{m}\right)-\operatorname{det}\left(\boldsymbol{F}_{m}^{\dagger} \boldsymbol{F}_{m}\right)=0$

where $\operatorname{Tr}_{2}\left(\boldsymbol{F}_{m}^{\dagger} \boldsymbol{F}_{m}\right)$ equals

$\sum_{k_{1}=1}^{3}\left(\sum_{j_{2}=2}^{J} \sum_{j_{1}=1}^{j_{2}-1} \frac{\Delta \ell_{k_{2} k_{3} j_{1} j_{2}}^{2}+2 L_{k_{2} k_{3} j_{1} j_{2}}\left(1-\cos 2 \pi \frac{m}{N} \delta_{k_{2} k_{3} j_{1} j_{2}}\right)}{\sigma_{j_{1}, m}^{2} \sigma_{j_{2}, m}^{2}}\right)$

with $k_{2}=k_{1}+1$ modulo 3 , and $k_{3}=k_{1}+2$ modulo 3 the two companion stars of $k_{1}$. Expression (16) is the sum of the determinants for the three subsystems with two-components. It is convenient and sufficient to solve this third-degree equation in $\omega^{2}$ numerically to obtain the condition number. Nevertheless, the analytical computation of the determinant adds insight in the discussion of ill-conditioned data sets of triple systems.

The determinant for the three-component system is

$$
\begin{aligned}
& \operatorname{det}\left(\boldsymbol{F}_{m}^{\dagger} \boldsymbol{F}_{m}\right)=\sum_{\left(j_{1}<j_{2}<j_{3}\right) \epsilon(1, \ldots, J)} \frac{\left(\sum_{k_{1}=1}^{3} \ell_{k_{1} j_{1}} \Delta \ell_{k_{2} k_{3} j_{2} j_{3}}\right)^{2}}{\sigma_{j_{1}, m}^{2} \sigma_{j_{2}, m}^{2} \sigma_{j_{3}, m}^{2}} \\
& +2 \sum_{k_{1}=1}^{3} \sum_{j_{1} \neq j_{2}, j_{3}} \frac{\ell_{k_{1} j_{1}}^{2}}{\sigma_{j_{1}, m}^{2}} \sum_{j_{3}=2}^{J} \sum_{j_{2}=1}^{j_{3}-1} \frac{L_{k_{2} k_{3} j_{2} j_{3}}\left(1-\cos 2 \pi \frac{m}{N} \delta_{k_{2} k_{3} j_{2} j_{3}}\right)}{\sigma_{j_{2}, m}^{2} \sigma_{j_{3}, m}^{2}} \\
& -2 \sum_{j_{1} \neq j_{2}, j_{3}} \sum_{j_{2} \neq j_{3}} \sum_{j_{3}=1}^{J} \frac{L_{123 j_{1} j_{2} j_{3}}\left(1-\cos 2 \pi \frac{m}{N} \delta_{123 j_{1} j_{2} j_{3}}\right)}{\sigma_{j_{1}, m}^{2} \sigma_{j_{2}, m}^{2} \sigma_{j_{3}, m}^{2}}
\end{aligned}
$$

with

$$
\begin{aligned}
L_{123 j_{1} j_{2} j_{3}} & =\ell_{1 j_{2}} \ell_{1 j_{1}} \ell_{2 j_{1}} \ell_{2 j_{3}} \ell_{3 j_{3}} \ell_{3 j_{1}} \\
\delta_{123 j_{1} j_{2} j_{3}} & =\left(\beta_{2 j_{1}}-\beta_{1 j_{1}}\right)+\left(\beta_{1 j_{2}}-\beta_{3 j_{2}}\right)+\left(\beta_{3 j_{3}}-\beta_{2 j_{3}}\right) \\
& =\left(\beta_{1 j_{2}}-\beta_{1 j_{1}}\right)+\left(\beta_{2 j_{1}}-\beta_{2 j_{3}}\right)+\left(\beta_{3 j_{3}}-\beta_{3 j_{2}}\right)
\end{aligned}
$$

and $\Delta \ell_{k_{2} k_{3} j_{2} j_{3}}, L_{k_{2} k_{3} j_{2} j_{3}}$ and $\delta_{k_{2} k_{3} j_{2} j_{3}}$ given by Eqs. (11) (the nonsimplified form on the first line) till (13). The following relations exist between the different relative velocities in the expression of the determinant:

$$
\begin{aligned}
\delta_{k_{1} k_{2} j_{1} j_{2}} & =\delta_{k_{1} k_{3} j_{1} j_{2}}+\delta_{k_{3} k_{2} j_{1} j_{2}} \quad\left(k_{1} \neq k_{2} \neq k_{3}\right) \\
\delta_{123 j_{1} j_{2} j_{3}} & =\delta_{13 j_{2} j_{3}}+\delta_{12 j_{3} j_{1}} \\
& =\delta_{12 j_{1} j_{2}}+\delta_{23 j_{2} j_{3}} \\
& =\delta_{23 j_{3} j_{1}}+\delta_{13 j_{1} j_{2}} .
\end{aligned}
$$

The outer sum in the first term of Eq. (17) cycles over all choices of three epochs out of the $J$ epochs with observations. Using the condition $\sum_{k=1}^{3} \ell_{k j}=1$, the inner sum over the three components simplifies to reduce the first line of Eq. (17) to

$\sum_{\left(j_{1}<j_{2}<j_{3}\right) \epsilon(1, \ldots, J)} \frac{\left(\Delta \ell_{k_{1} k_{2} j_{1} j_{2}}+\Delta \ell_{k_{1} k_{2} j_{2} j_{3}}+\Delta \ell_{k_{1} k_{2} j_{3} j_{1}}\right)^{2}}{\sigma_{j_{1}, m}^{2} \sigma_{j_{2}, m}^{2} \sigma_{j_{3}, m}^{2}}$

where $k_{1}, k_{2}$ stands for any choice of two of the three components.

\subsubsection{Fourier mode $m=0$}

The elements of the design matrix for the $m=0$ mode do not depend on the relative Doppler shifts, but only on the $\ell_{k j}$ and the $\sigma_{j, m}$, and the expression for the determinant reduces to a sum of squares for any number of components $K$ :

$\operatorname{det}\left(\boldsymbol{F}_{0}^{\dagger} \boldsymbol{F}_{0}\right)=\sum_{\left(j_{l}<j_{2}<\ldots<j_{K}\right) \epsilon(1, \ldots, J)} \operatorname{det}^{2}\left(\boldsymbol{F}_{0\left(j_{1}, j_{2}, \ldots, j_{k}\right)}^{\prime}\right)$.

Equations (11) and (18) show how this general expression can be simplified, for $K=2$ and $K=3$, respectively. The design matrix is singular if and only if there is at least one couple of components $\left(k_{1}, k_{2}\right)$ with a mutual light-ratio $r_{k_{1} k_{2} j}=\ell_{k_{1} j} / \ell_{k_{2} j}$ that does not vary over the input spectra (does not depend on $j$ ), since then the elements in the columns $k_{1}$ and $k_{2}$ of the normal matrix are proportional. If none of the $r_{k_{1} k_{2} j}$ depends on $j$, then $K-1$ singular values will be zero. The singularity implies that a wavelength-independent change in level can be added to the intrinsic spectrum of one component, if a corresponding change is applied to the intrinsic spectrum (spectra) of the component(s) having a constant mutual light-ratio relative to the first one. The set of solutions has been described in Sect. 2.

However, with a significantly varying, mutual light-ratio, the solution of the zero-mode equation guarantees the proper normalisation of the component spectra, and thus the proper reconstruction of the total line-blocking coefficient over the observed wavelength region. Even if a subset of all components has a constant mutual light-ratio, the remaining components will be normalised correctly if the zero-mode equation is solved using SVD. The particular case of eclipsing binary stars, where few observed spectra may correspond to a very different mutual lightratio, deserves further comments.

Figure 3 illustrates how the inclusion of a spectrum in eclipse eliminates the singularity from the zero-mode equation, and how the use of a larger weight for such spectrum increases 


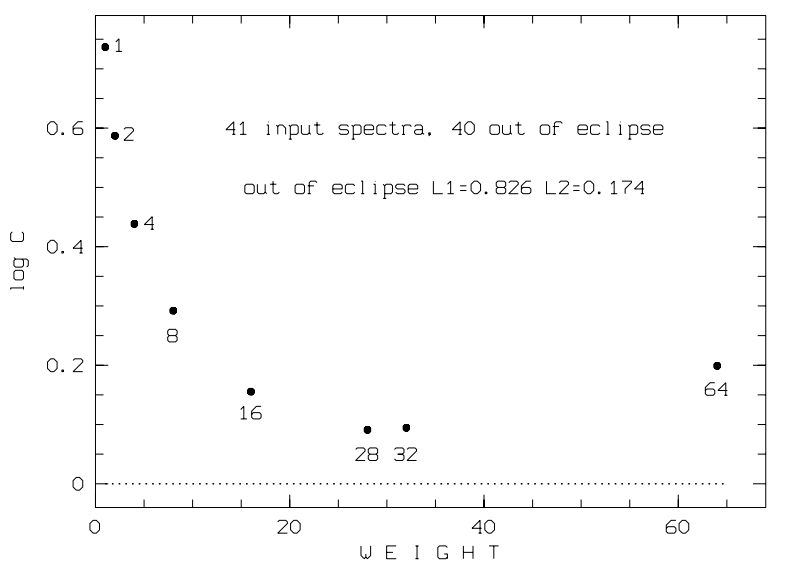

Fig. 3. The improvement in condition number for a data set of 41 spectra well-distributed over the orbital phase and including one spectrum in totality of an eclipse. The data set is constructed from an observational data set for RV Crt, but only considering the close binary in this triple system. Out of eclipse, the star which is invisible in mid-eclipse has $82.6 \%$ of the total light. The condition number improves by almost a factor of 5 when counting the eclipse spectrum with a weight in a broad interval around 28.

the regularity of the set of equations. Indeed, when weights are assigned relative to the random noise in the spectra and when most spectra are taken out of eclipse, then the information present in the varying dilution of the spectral lines during eclipse is taken into acount with low weight. An argument against giving more weight to spectra taken in eclipse (unless the eclipse is total) is that the shape of the line-profiles in the spectrum of the eclipsed star changes when part of its disk is eclipsed. The interesting possibility in the Fourier-approach is, that one can assign a large weight to the eclipse spectra for low-frequency modes, and low or null weight for high-frequency modes. In this way, the dilution of the spectral lines during eclipse is used to stabilise the solution for the low-frequency modes, while it does not influence the line profiles. This is the basic reason that we consider weights that depend on the Fourier mode $m$.

As an example, we compute the optimal weight $w_{\text {opt }}$ to be associated with a single total-eclipse spectrum of a binary in a set of $J$ equal-quality spectra with no light variability out of eclipse. In this case, $\ell_{1 j}=1$ for $j=1$ and $\sigma_{1}^{-2}=w \sigma_{j>1}^{-2}$ for $m=0$. Since $C_{0}$ is a monotonic function of $z_{0}$ (Eq. (15)), it suffices to require that the derivative of $z_{0}$ to $w$ is zero. With

$z_{0}=\frac{w+(J-1)\left(\ell_{1}^{2}+\ell_{2}^{2}\right)}{2 \ell_{2} \sqrt{J-1} \sqrt{w}}$

one finds

$w_{\mathrm{opt}}=(J-1)\left(\ell_{1}^{2}+\ell_{2}^{2}\right)$

and with this weight

$C_{0, \mathrm{opt}}=\frac{\ell_{1}}{\ell_{2}}+\sqrt{1+\frac{\ell_{1}^{2}}{\ell_{2}^{2}}}$.

Notice that the "optimal" condition number is independent of the size of the data set, but will vary with wavelength if the stars have a different flux distribution. Since

$z_{0}(w=1)=\frac{1+w_{\mathrm{opt}}}{2 \sqrt{w_{\mathrm{opt}}}} z_{0, \mathrm{opt}} \quad$ and $\quad w_{\mathrm{opt}} \propto J-1$,

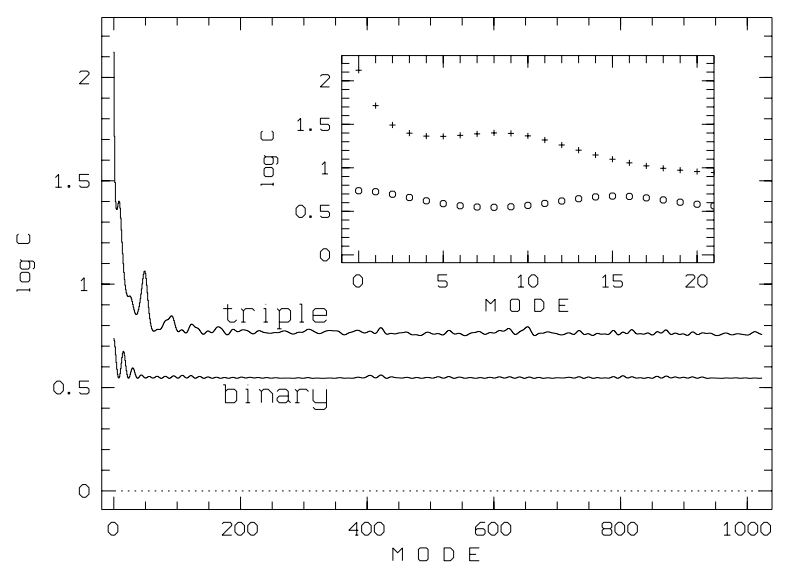

Fig. 4. Deterioration of the condition number by a third companion to an eclipsing close binary, when the relative light ratio of the third star to one of the close-binary components varies only because of the nonspherical shape of the latter component. The example refers to the data set of RV Crt used also in Fig. 3 with unit weight for the spectrum in eclipse. The condition number for the binary deviates from unity because of $\frac{\ell_{1}}{\ell_{2}} \approx 4.75$ out of eclipse. The observed region is 2048 pixels wide, $\approx 26\left(K_{1}+K_{2}\right)$. The condition number for the lowest modes is shown in detail in the smaller window.

the use of the "optimal" weight has a larger impact on the condition number for a data set with a smaller amount of eclipse spectra.

It is straightforward to generalize Eqs. (19) and (20) to the case of a partial eclipse,

$$
\begin{aligned}
w_{\mathrm{opt}} & =\frac{(J-1)\left(\ell_{1}^{2}+\ell_{2}^{2}\right)}{\ell_{1 \mathrm{e}}^{2}+\ell_{2 \mathrm{e}}^{2}} \\
C_{0, \mathrm{opt}} & =\frac{\frac{\ell_{1}}{\ell_{2}} \frac{\ell_{1 \mathrm{e}}}{\ell_{2 \mathrm{e}}}+1+\sqrt{\left(1+\frac{\ell_{1}}{\ell_{2}}\right)\left(1+\frac{\ell_{1 \mathrm{e}}}{\ell_{2 \mathrm{e}}}\right)}}{\left|\frac{\ell_{1 \mathrm{e}}}{\ell_{2 \mathrm{e}}}-\frac{\ell_{1}}{\ell_{2}}\right|}
\end{aligned}
$$

where the index "e" was added for the light factors in partial eclipse. A larger weight is needed than in the case of a total eclipse $\left(\ell_{1 \mathrm{e}}^{2}+\ell_{2 \mathrm{e}}^{2}<1\right)$, and the improvement of the condition number is smaller. Shallow eclipses $\left(\ell_{1 \mathrm{e}} / \ell_{2 \mathrm{e}} \approx \ell_{1} / \ell_{2}\right)$ have no significant effect. The set of equations will remain illconditioned. Shallow, in this sense, means that the varying dilution effect on the spectral lines is not larger than the uncertainty in placing the continuum level on the observed spectra. Similarly, intrinsic stellar light-variability in non-eclipsing binaries only helps in solving the zero-mode equation if the amplitude of the light variations is large.

\subsubsection{Very low-frequency Fourier modes, $m \neq 0$}

The determinant of non-zero modes contains two types of terms: the terms depending on the light-ratios only (which appear also in the $m=0$ case), and an additional non-negative contribution depending on light-ratios and relative velocities. Hence, if the zero-mode is well-conditioned, all higher modes are also well-conditioned since their determinant is not smaller than for $m=0$. The discussion below is thus only relevant for systems with light ratios that do not vary significantly. Figure 4 shows the increase in condition number due to the inclusion of a third, wide component contributing significantly to the total light $(\approx 45 \%)$ with a close binary where only one spectrum was obtained in totality of the deeper eclipse. The third component, although 
dominating the observed spectrum, destabilizes the lowfrequency solution because its light ratio relative to the noneclipsed component of the close binary only varies slightly owing to the ellipsoidal shape of the latter component. In the two lowest modes, the condition number is enhanced by a factor of 24 and 10, respectively.

If $\cos 2 \pi \frac{m}{N} \delta_{12 j_{1} j_{2}} \approx 1$ for all $\left(j_{1}, j_{2}\right)$ in the case $K=2$ or $\cos 2 \pi \frac{m}{N} \delta_{k_{1} k_{2} j_{1} j_{2}} \approx 1$ for all $\left(j_{1}, j_{2}\right)$ and all $\left(k_{1}, k_{2}\right)$ in the case $K=3$, then the set of equations for specific non-zero modes may be ill-conditioned. This can be evaluated quantitatively through calculation of the condition number. Low $\frac{m}{N}$ (lowfrequency Fourier modes) and small relative velocities are unfavourable factors. In such a case, application of SVD is the recommended option to obtain a robust solution. This solution may deviate from the true solution and introduce a spurious signature in component spectra. A spurious signature at low frequency is likely to appear in component spectra reconstructed over a wavelength interval much larger than the total range in relative Doppler shifts between the components. However, these spurious signatures in the different components are strictly linked together, such that their contribution cancels when combining the component spectra to reproduce the observed spectra. Removal of spurious signatures has to take into account this coupling. Methods "re-normalising" the component spectra independently (e.g., Fitzpatrick et al. 2002) produce normalised component spectra with a worse goodness-of-fit.

Identification of the ill-conditioned modes ( $m$-values) thus defines the shape of possible spurious features and this knowledge can be combined with the identification of apparent continuum windows to obtain an appropriate normalisation if the SVD solution did provide an unsatisfactory result. In practice, it may happen that none of the allowed shapes offers a satisfactory result. This indicates that either the model (Eq. (1)) is biased or that the observed spectra are biased (e.g. incorrect normalisation). The latter is the subject of Sect. 5 and the description of a practical normalisation procedure in ill-conditioned cases will be discussed thereafter (Sect. 6).

\subsubsection{Higher Fourier modes}

The set of equations for higher Fourier modes is ill-conditioned in exceptional cases, namely for very specific distributions of the relative velocities, which are more easily fulfilled for small sets of observed spectra and when the velocities are roundedoff to the nearest integer bin. Figure 5 shows artificial data sets with some ill-conditioned high-frequency Fourier mode(s). The shape of the component spectra is identical and represents a sharp-lined B1 V spectrum ( $\approx 4580-4670 \AA)$. The same sets of artificial data are extensively used in Sect. 5. The input spectra are constructed for 16 orbital phases, at velocities $-K_{1},-\frac{11}{12} K_{1}$, $-\frac{3}{4} K_{1},-\frac{7}{12} K_{1},-\frac{1}{2} K_{1},-\frac{5}{12} K_{1},-\frac{1}{3} K_{1},-\frac{1}{12} K_{1}, 0, \frac{1}{6} K_{1}, \frac{1}{4} K_{1}, \frac{5}{12} K_{1}$, $\frac{2}{3} K_{1}, \frac{5}{6} K_{1}$ and $\frac{11}{12} K_{1}, K_{1}$ to mimic a good coverage of the orbit.

The origin of the indeterminacies lies with the $\mid\left(\beta_{2 j}-\beta_{1 j}\right)-$ $\left(\beta_{2 j^{\prime}}-\beta_{1 j^{\prime}}\right) \mid$ for all couples of orbital phases (index $\left.j, j^{\prime}\right)$ being multiples of 2,3 or 4 respectively (using $K_{1}=12, K_{2}=$ $12,24,36)$ and the number of bins in the reconstructed spectra (672) being a multiple of 2, 3 or 4 . Solving with SVD, the amplitude of the high-frequency patterns is much lower than for low-frequency modes and actually of little practical interest. The reason for this is explained in Appendix A (Sect. A.4).

Finally, we notice that the equations for all Fourier modes are ill-conditioned when one of the components is very faint in all spectra. This is easily illustrated and quantified for the case
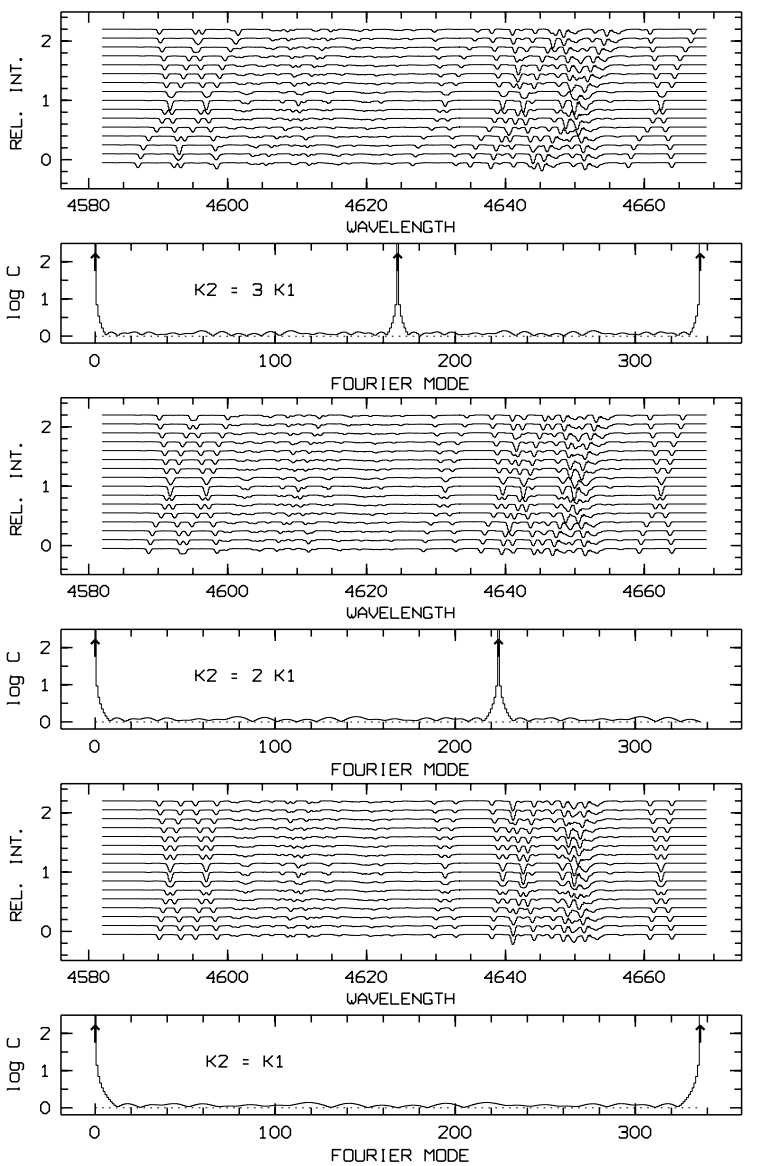

Fig. 5. Artificial data sets (16 spectra, 672 bins) leading to illdetermined Fourier modes at $m=0$, and $|m|=336$ and/or $|m|=224$ and/or $|m|=168$ (arrows indicate $C=\infty$ ). Also neighbouring modes are determined with less precision. The three data sets differ only by the amplitude $K_{2}$ of the Doppler velocities, as indicated in the panels showing the logarithm of the condition number. The corresponding input data sets are shown above each of the $\log C$-panels.

of a binary star: assuming a good orbital coverage with equalquality spectra, the expectation value for the sum of cosine terms in Eq. (10) is zero, and the expectation value for the determinant in the constant-light case is $J(J-1) \ell_{1}^{2} \ell_{2}^{2} \sigma_{m}^{-4}$. Since for $\ell_{1}+\ell_{2}=1$

$\ell_{1} \ell_{2}=\frac{1}{2}\left(1+\left(\ell_{1}-\ell_{2}\right)\right) \frac{1}{2}\left(1-\left(\ell_{1}-\ell_{2}\right)\right)=\frac{1}{4}\left(1-\left(\ell_{1}-\ell_{2}\right)^{2}\right)$

the expectation value for $z_{m}$ is

$z_{m} \rightarrow \sqrt{\frac{J}{J-1}} \frac{1+\left(\ell_{1}-\ell_{2}\right)^{2}}{1-\left(\ell_{1}-\ell_{2}\right)^{2}}$

and, approximating $J /(J-1)$ by unity, simple algebra leads to

$C_{m} \approx \ell_{1} / \ell_{2}$.

Hence, for a binary with equal brightness components, the condition numbers approach unity. In the other extreme, with a vanishingly weak secondary, all Fourier components are extremely ill-conditioned. This extreme expresses simply that a component cannot be reconstructed if the observed spectra do not contain information about it. Equation (22) quantifies that the spectral separation for each component of a (non-eclipsing) binary star degrades inversely proportional to its fractional light contribution. The weaker the component, the worse the set of equations is constrained. This reflects, in the first place, the difficulty to 

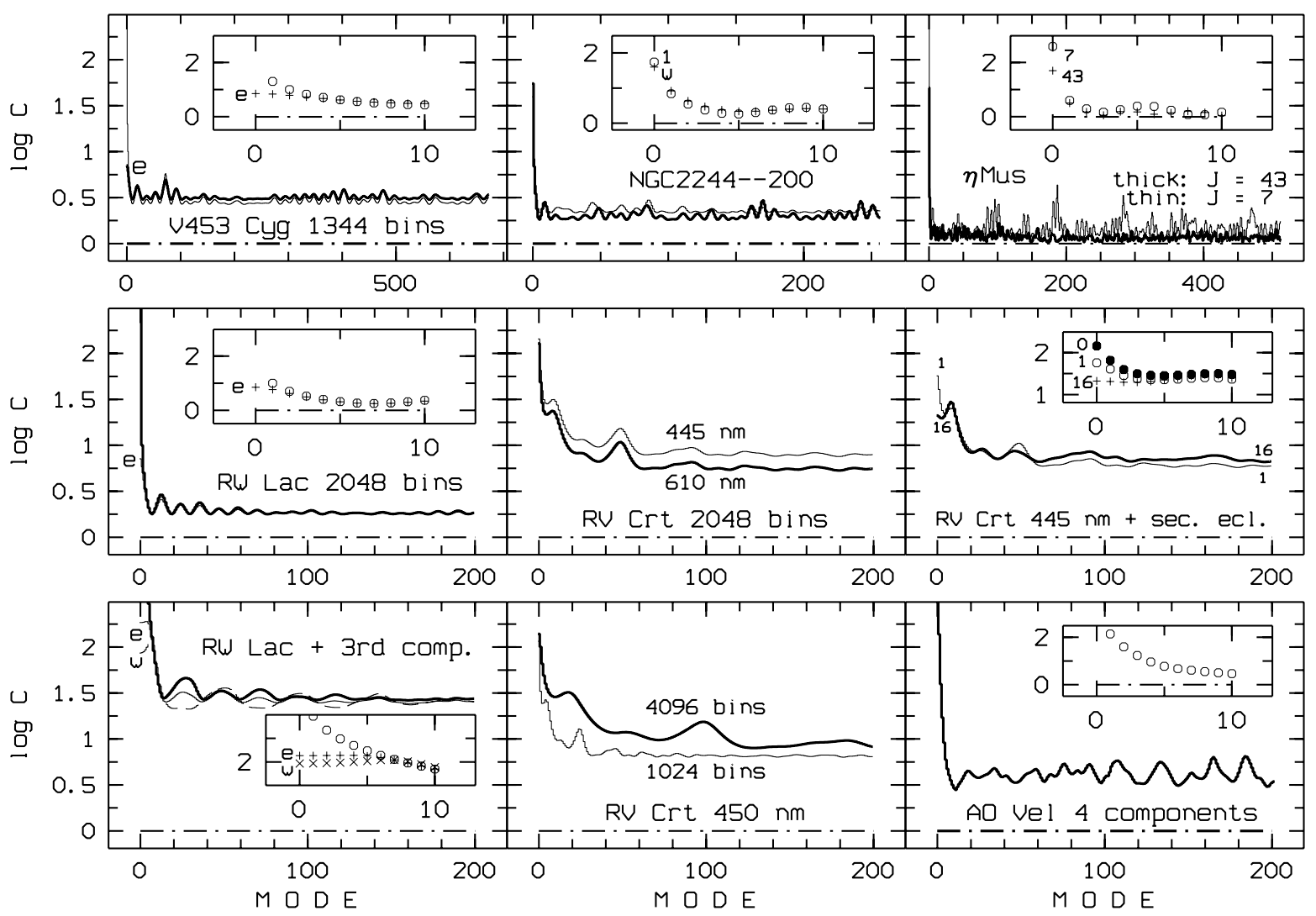

Fig. 6. Condition numbers for real data sets (see text). Notice only part of the Fourier modes are shown (the lower-frequency modes) in the panels on RW Lac and RV Crt, and that the panels showing the $m=0-10$ modes have different scales on the vertical axis.

reconstruct the spectrum of the faintest component. While low random noise (or, equivalently, a large number of observed spectra) is a prerequisite for the reconstruction of weak components, and will set in practise often a more stringent limitation than the condition number, the latter indicates that beyond a certain light ratio even low random noise may be insufficient to reconstruct a faint component.

\subsection{The velocity-space case}

If a problem is ill-determined in Fourier space, it is also illdetermined in velocity space. The reverse is not necessarily true (Appendix A), but the "gain" in the Fourier approach is a consequence of the additional periodicity requirement on the spectra, which is not based on physics. The "gain" is thus illusory.

Since the singular values in the case of the velocity space refer to a large set of coupled equations, it is less easy to predict the shape of the spurious component theoretically, as compared to the singular values associated directly with different Fourier modes. However, this point must be evaluated in the context that the presence of bias in the input spectra (Sect. 5) may invalidate the predicted shape in the Fourier approach.

\subsection{Applications}

We computed condition numbers for a number of observed data sets available in the literature to illustrate their usefulness in practice. Figure 6 summarizes the results in eight panels.

The upper-left panel deals with the data set of 8 spectra of the early-B type eclipsing binary V453 Cygni analysed by Simon \& Sturm (1994) in their paper introducing the velocity-space disentangling technique. The condition numbers $C_{m}$ are all larger than $\ell_{1} / \ell_{2} \approx 2.7$. Despite the low number of spectra, the equations for all modes are well-determined if the spectrum in total eclipse is included (thick line, "+" symbol in Fig. 6), or if the light ratio is imposed by external knowledge. Otherwise (thin line and "o" symbol), the $m=0$ mode is undetermined and larger undulations of quasi-sinusoidal shape might be seen.

The middle panel in the upper row deals with another early-B binary, NGC 2244-\#200, this time with very partial eclipses and $\ell_{1} / \ell_{2} \approx 1.9$ near $4600 \AA$. The data set of 12 spectra analysed by Hensberge et al. (2000) to reconstruct the spectra and to derive the orbit includes two spectra in the middle of one of the partial eclipses. Weights were assigned to the spectra (thick line, "+") following signal-to-noise ratio such that the spectra in eclipse received together only $7 \%$ of the total weight. The thin line and the open circles refer to the case without weight. The weights had no significant influence on the condition numbers, but, as shown in Fig. 3, assigning a much larger weight to the eclipse spectra in the solution of the $m=0$ and $m=1$ modes would reduce the undulations removed by the authors applying a "corridor" procedure as described in Sect. 6.

The upper-right panel in this row deals with two data sets for $\eta$ Muscae used by Bakis et al. (2007) to reconstruct the spectra of two very similar late-B type components with very partial eclipses and to derive the orbit. One data set includes 43 spectra of which several were in partial eclipse (thick line, “+”). The newer data set in a different wavelength region includes only 7 spectra (thin line, "o"), one of which is in a partial eclipse. Both $\log C$-curves are noisier because measured radial velocities were used rather than those predicted by the orbit. It is 
obvious that the larger data set, well-distributed over the velocity range covered by the orbit, reduces the structure in the $\log C$-data significantly. While the use of the spectra in partial eclipse, but out of mid-eclipse, helps in constraining the lowest modes (and larger weights would have been beneficial to modes $m=0,1$ ), it might be wise not to use such spectra for the solution of the higher modes since the line profiles in those orbital phases are different.

Below, the mid- and lower-left panel deal with the data set for RW Lacertae analysed by Lacy et al. (2005) with the twodimensional cross-correlation technique developed by Zucker \& Mazeh (1994). It concerns an eclipsing late-type binary (G5 + G7) with $\ell_{1} / \ell_{2} \approx 1.8$ near $5200 \AA$, for which 36 spectra were collected, almost exclusively at orbital phases corresponding to large Doppler shifts $\left(v_{k j}>K_{k} / 2\right)$. The lack of spectra at lower velocities is reflected in small oscillations of $\log C$ for the first $\approx 80$ modes. Only the first 200 of 1025 modes are shown, because further on the curve is flat. In the mid-row panel, the thin line (almost indistinguishable from the thick one) and the "+" indicate the beneficial effect that two spectra with unit weight, taken at each mid-eclipse would have: the condition number of mode $m=0$ is reduced from a value above $10^{3}$ (even while taking into account the very small light variations out of eclipse) to below 10 (proper weighting would bring it down even more), allowing a spectroscopic determination of the light ratio. In fact, Lacy et al. (2005) suspect that the system contains a third component contributing roughly $2.5 \%$ to the total light. The lowerrow panel shows how much such a faint companion enhances the condition number of the equations. Especially when no eclipse spectra are available (thick line, "o"), various low-frequency modes are destabilised. The eclipse spectra defined above, in which the faint component has a larger contribution to the total light, reduce the effect (thin line and "+" refer to unit weight, dashed line and " $x$ " to a ten times larger weight for the eclipse spectra), but $\log C$ remains one order of magnitude larger than in the binary case for all modes. A complete spectroscopic study of this system should be undertaken only when some spectra in eclipse are available.

Three of the four remaining panels concern the triple system RV Crateris (Hensberge et al. 2007; Vaz et al. 2008), consisting of an eclipsing binary (late-F + late-G) representing about half of the total light and a distant, sharp-lined late-F companion. The faint component of the close binary contributes less than $10 \%$ to the total light below $6000 \AA$. The data set includes 41 spectra, of which one was obtained when the brighter component of the close binary was totally eclipsed. Twenty-five different wavelength intervals were reconstructed, but only those with the most significant contribution of the fainter component were used for the orbit determination. This case serves to show the influence of a number of different parameters: the lower light contribution of the faintest star at blue wavelengths enhances the condition number for all modes, but not very significantly. A large wavelength range can be used. Taking a four times larger wavelength region around the same central wavelength results in any structure in the $\log C$-curves being moved to four times higher modes (compare the bump near $m=100$ in the 4096bin case to that near $m=25$ for the 1024-bin case). Finally, the righthand panel in the middle row shows the effect of adding one spectrum in the middle of the very faint secondary eclipse, with weight unity (thin line, "o") or weight 16 (thick line, "+"). For comparison the result for the first ten modes without this secondary-eclipse spectrum (weight 0 ) is shown with filled discs. It shows that a spectrum in the secondary eclipse is useful for stabilising the solution of low Fourier modes, and thus reducing the undulations seen in Fig. 11, although the original perception when planning the observations was that the line profiles at the time of these ring-like eclipse would exclude any use. (Evidently, it should not be used for any but the lowest modes.)

Finally, the lower right panel deals with the only fourcomponent system with reconstructed spectra (González et al. 2006) from a small data set of 11 spectra, AO Velorum, analysed with a tomographic technique. The stars contribute $40 \%$, $28 \%, 16 \%$ and $16 \%$ to the total light. Lacking sufficent indications in the paper, we took the light ratio as constant in time, although one of the stars is eclipsing and three spectra appear to be taken with one component partially in eclipse. Nevertheless, the computation shows that all except the lowest modes have very acceptable condition numbers, indicating that it is not too unreasonable to reconstruct the spectra from such complex system from a small data set. The most adverse effects are probably confined to the lowest modes, with modes up to $m=4$ having condition numbers larger than 10 . This is interpreted as a larger sensitivity of higher-multiplicity systems to undulations with a more complex pattern being superposed on the continua of the component spectra.

\section{Biased input spectra}

The input data for the spectrum reconstruction analysis have undergone a data reduction process aiming at the removal of complex instrumental effects, as fringing, contributions of stray light, intensity modulation through the spectral orders, etc. Imperfect data reduction (and imperfect detectors) may generate spurious features in the observed spectra at very different frequencies. A particularly difficult issue in echelle spectroscopy is the combination of data observed in different spectral orders. As a rule, the standard pipeline data reduction software implemented at the telescopes do not offer consistent data at the same wavelengths in different spectral orders, in terms of low-frequency changes of the flux with wavelength. They may merge the inconsistent information in a global spectrum (which then contains instrumental order-merging bias) or they may leave it to the researcher to determine the stellar continuum in each spectral order separately, a quite nonrobust procedure.

Even when these types of pitfalls are successfully circumvented, the normalisation of the observed spectrum is likely more complex than for single stars. Not only are there spectral features of more than one component, the features also move in time. As a consequence, the position and the number of continuum windows - if any are present - will change in accordance with orbital phase. At some level, this will introduce phasecorrelated bias in the normalisation of the spectra. This bias will be transferred by the purely mathematical separation algorithm to the output component spectra. The basic characteristics of the bias progression constitute the subject of this section.

Our main concern is the phase-correlated bias, since this leads to large bias in the output spectra. Nevertheless, some specific examples of static bias will be discussed, without any attempt to exhaust the subject. Our study is based on artificial, noise-free input spectra. They serve our purpose to illustrate under which conditions bias is amplified in the output spectra. While random noise complicates the determination of the observed continuum and increases the uncertainty in it, it is unlikely to increase the correlation of bias with orbital phase, except when the noise level correlates itself with orbital phase. 


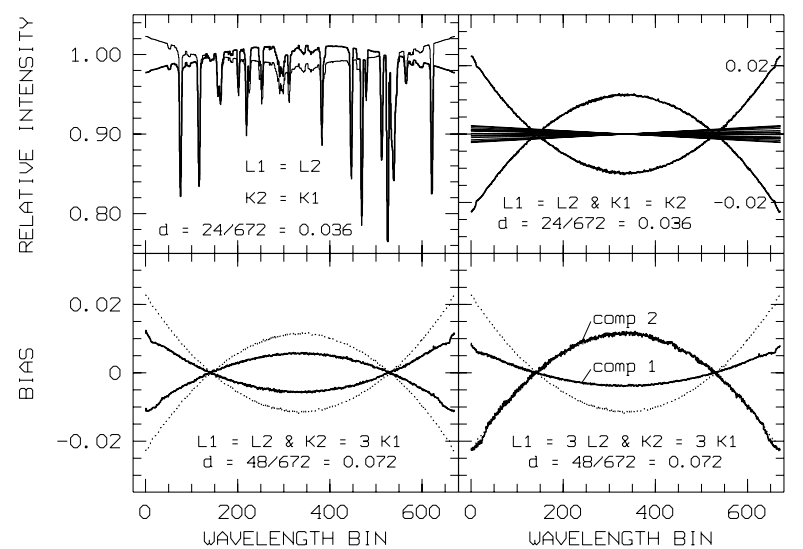

Fig. 7. Bias progression during the reconstruction of component spectra in the case of a continuum gradient error that correlates strictly with orbital phase. The upper panels show the reconstructed spectra (left), and the bias in the 16 input (thin lines) and the 2 reconstructed component spectra (thick lines) in units of continuum intensity for the case of identical components, with the extreme difference in Doppler shifts between the components equal to $3.6 \%$ of the length of the reconstructed wavelength interval. The lower panels show the inverse proportionality of the amplitude of the bias with the relative Doppler length $d$ (compare left lower panel to right upper panel) and the inverse proportionality with the fractional light ratio $\ell_{j}^{-1}$ (compare right lower panel with left lower panel).The dotted lines in the lower panels refer to the identicalcomponents case shown in the right upper panel.

\subsection{Bias related to orbital phase}

To separate the progression of the phase-related bias from other factors, all examples are built from the same, noiseless component spectra of a binary star. Without loss of generality in the conclusions, the spectra of the two components are taken to be identical (see Fig. 5), the covered wavelength range $c \Delta \ln \lambda$ and its discretisation in $N$ bins are always the same and the velocity amplitude $K_{1}$ fixed. However, we vary the type of bias applied to the continuum, the velocity-amplitude ratio $K_{1} / K_{2}$ and thus also the relative Doppler length $d=\left(K_{1}+K_{2}\right) /(c \Delta \ln \lambda)$ in units of the size of the spectral interval, and the light ratio $\ell_{1} / \ell_{2}$. The light-ratio is time-independent except for the discussion of one specific case, wherein a mid-eclipse spectrum is included.

The bias $\epsilon_{j}(\ln \lambda)$ applied to the continuum has an amplitude depending on wavelength and orbital phase,

$y_{j}(\ln \lambda) \rightarrow y_{j}^{\prime}(\ln \lambda)=\left(1+\epsilon_{j}(\ln \lambda)\right) y_{j}(\ln \lambda)$.

We took the continuum to be unbiased at the velocity of the system since this should be the spectrum that is easiest to normalise, and bias increased proportional with the Doppler shifts to a maximum of 0.0025 ( $0.25 \%$ of the flux in the continuum). As a function of wavelength, we represented the bias in different ways: a sinusoidal function of $\ln \lambda$, with period equal to or smaller than the length of the spectrum; a slope superposed on the continuum level; or a constant offset, with opposite sign at both sides of the centre, linearly connected around the centre. These different types of bias, as a function of wavelength, represent in a schematic way continuum offsets (a risk when merging spectral orders from an echelle spectrograph), continuum slope errors, and wavy patterns due to the use of pseudo-continuum windows.

The resulting bias in the reconstructed spectra is shown in Figs. 7, 8. The first figure concentrates on the influence of light ratio, velocity-amplitude ratio, and relative size of the interval in the case of a local error in the gradient of the continuum. A similar figure for a sinusoidal disturbance of the continuum can

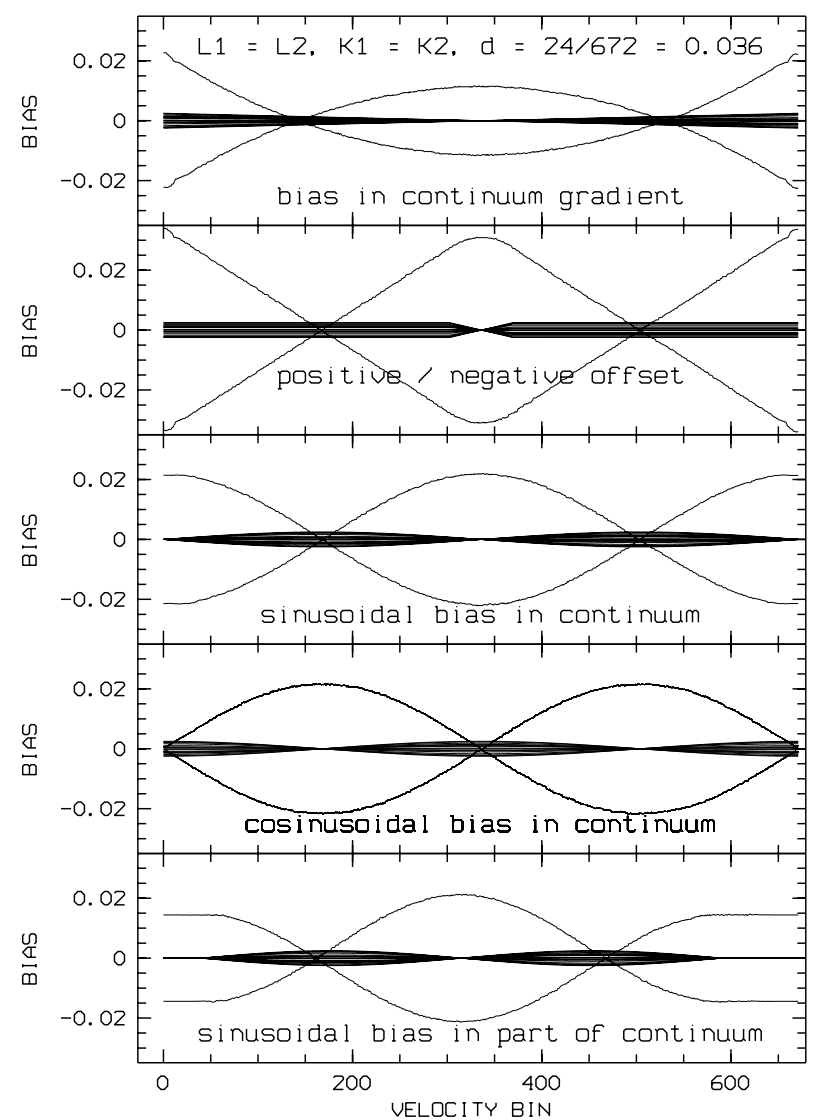

Fig. 8. Bias progression during the reconstruction of component spectra. Different panels refer to different bias patterns in the input spectra, all strictly correlated with orbital phase. All cases have identical light ratio, velocity amplitude ratio, relative Doppler length and orbital phase distribution of input spectra (see text and label in upper panel). The two higher-amplitude curves in each panel represent the bias in the reconstructed spectra, the bunch of 16 lower-amplitude curves represent the bias in the input spectra. The bias is expressed in units of continuum flux intensity.

be found in Hensberge \& Pavlovski (2007). The amplification of phase-correlated bias during the reconstruction process is obvious. Figure 8 concentrates on different types of bias as a function of wavelength. The bias seen in the reconstructed spectra is easily understood in the following way. Assume that the observed spectra $y_{j}$ contain an additive bias $\eta_{j} b(\ln \lambda)$, where $\eta_{j} \in[-1,+1]$ is proportional to the instantaneous radial velocity and $b(\ln \lambda)$ is a smooth function. In practice, the bias we applied is not additive (Eq. (23)), but it is a good approximation for small $b(\ln \lambda)$ except when many wavelength bins contain much less flux than the continuum. In order to reproduce this bias for arbitrary $\eta_{j}$ a bias $\pm B(\ln \lambda) / \ell_{k}$ in the reconstructed spectra is needed, since

$$
\begin{aligned}
\eta b(\ln \lambda) & =\ell_{1}\left[\frac{1}{\ell_{1}} B\left(\ln \lambda+\eta \frac{K_{1}}{c}\right)\right]+\ell_{2}\left[\frac{-1}{\ell_{2}} B\left(\ln \lambda-\eta \frac{K_{2}}{c}\right)\right] \\
& =\eta \frac{K_{1}+K_{2}}{c} \frac{\mathrm{d}}{\mathrm{d} \ln \lambda} B(\ln \lambda) .
\end{aligned}
$$

It immediately follows that

$B(\ln \lambda)=\frac{c}{K_{1}+K_{2}} \int^{\ln \lambda} b(x) \mathrm{d} x$,

i.e., the function $B$ describing the bias in the reconstructed spectra is the integral of the additive bias in the observed spectra. 


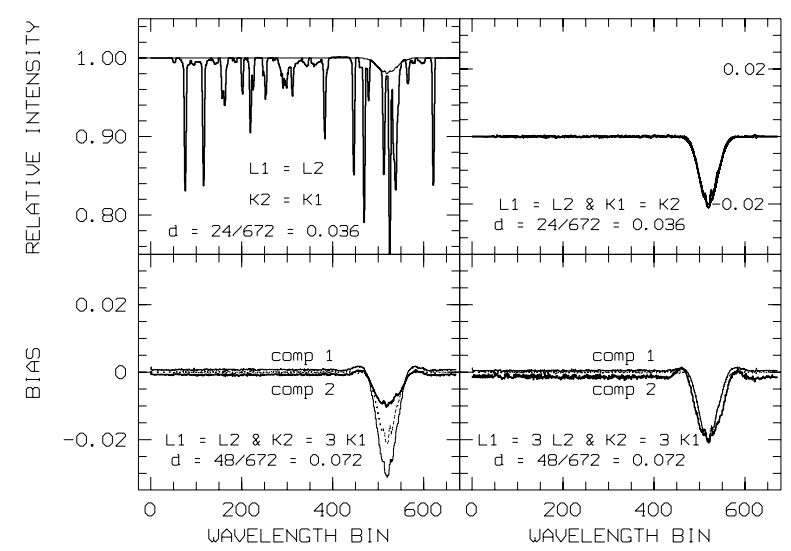

Fig. 9. Bias progression during the reconstruction of component spectra in the presence of a $2 \%$-deep static absorption feature (thin line in upper right panel) not belonging to the reconstructed components. The input spectra (except for the static feature), the parameters, and the lay-out of the figure are the same as in Fig. 7. The bias is expressed as the difference between the reconstructed spectra and the true stellar spectra.

Defining the variable $\xi=2\left(\ln \lambda-x_{0}\right) /(\Delta \ln \lambda)$, where $x_{0}$ is $\ln \lambda$ at the mid-point of the considered log-wavelength interval, one can also rewrite the above expression as

$B(\xi)-B(0)=\frac{c \Delta \ln \lambda}{2\left(K_{1}+K_{2}\right)} \int_{0}^{\xi} b\left(x^{\prime}\right) \mathrm{d} x^{\prime}=\frac{1}{2 d} \int_{0}^{\xi} b\left(x^{\prime}\right) \mathrm{d} x^{\prime}$.

This explains the proportionality of the amplitude of the bias in the reconstructed spectra to the length of the interval in Doppler units, $d^{-1}$, and the inverse proportionality to the light factors, as seen in Fig. 7. It also explains the parabolic shape of $B$ when $b$ is linear ("gradient" case), the linear shape of $B$ for the "offset" case for $b$ and the trigonometric shapes in the remaining three cases (Fig. 8). Equation (24) also predicts very well the observed amplitude of the bias in the reconstructed spectra.

We performed the tests shown thus far with on average bias zero in each input spectrum. Exactly such bias is likely to pass unnoticed since it cannot be detected by simply checking the consistency of the line blocking in the observed spectra. As expression (24) shows, in all such cases the bias $B$ has the same value at the edges of the reconstructed spectra. Exactly this feature leads to the same response for the velocity-space and Fourier-space algorithms. However, if the phase-correlated bias described by $b$ has non-zero average over the considered wavelength interval, the bias in the reconstructed spectra may be different for the two algorithms (Ilijić 2004).

\subsection{Bias fixed in velocity space}

In principle, blemishes due to the detector, imperfect flatfielding, unrecognized faint interstellar features, telluric lines or absorption due to a faint, undetected component can all contribute to (quasi)-static features in the observed spectra that are not included in the model described by Eq. (1). If the model includes the reconstruction of a static stellar component, then static features of any different origin are fully transferred to this component. Further discussion concerns models without static stellar component.

Figure 9 shows the results obtained by adding to the data set shown in the lower panels of Fig. 5 a weak static absorption feature, while the spectral lines of both reconstructed components show orbital Doppler shifts. A lower light contribution and a lower Doppler amplitude contribute to make the static feature

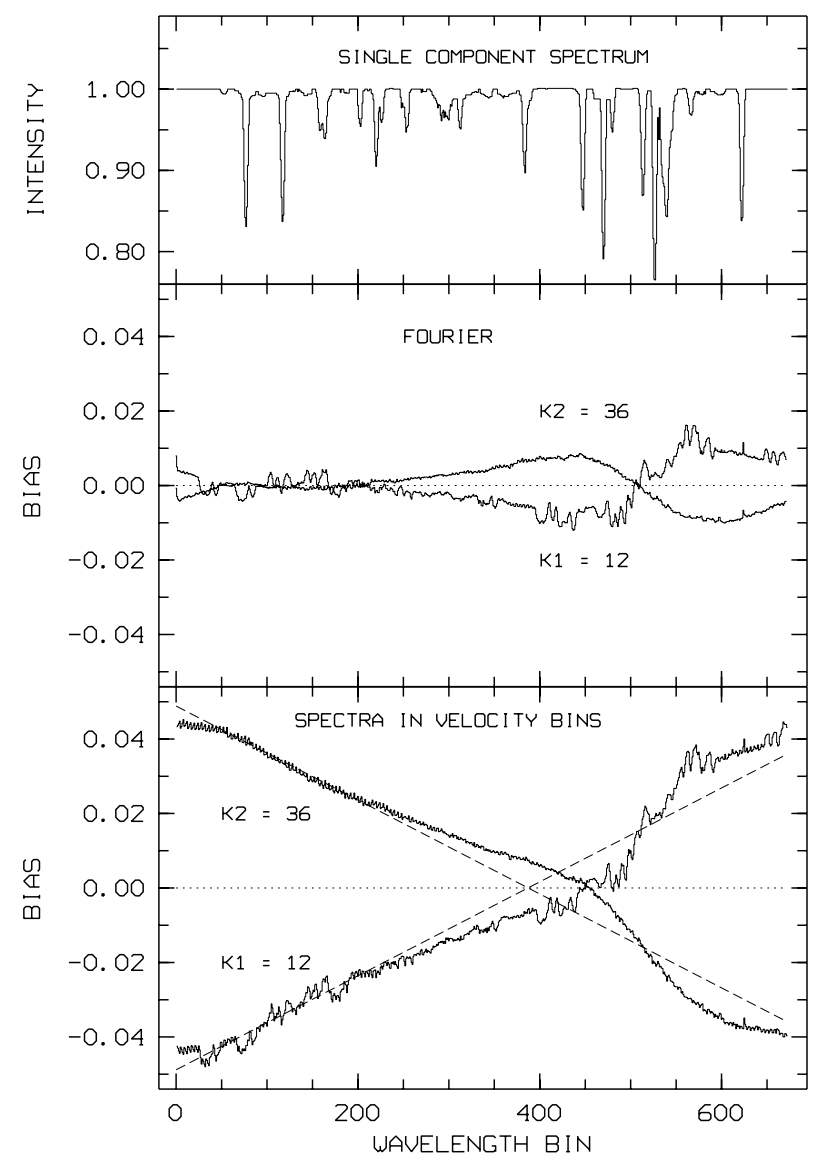

Fig. 10. Bias induced by line strength variability in one of the components (see text for details). The upper panel shows the true spectrum of a single component (full line) for $v=0$. The bias in the reconstructed spectra is shown in the middle panel (applying the Fourier technique) and the lower panel (applying the velocity-space technique). In the latter case, the reconstructed spectra were shifted by +0.04 and -0.04 in intensity to present the results within a smaller range of intensities.

more pronounced (and slightly deformed) in the reconstructed spectrum. In addition, small offsets of the reconstructed spectra appear over the whole wavelength region.

\subsection{Bias in line strengths}

Another example of bias would be unnoticed line strength variability in one of the components. If the variability correlates with the orbital phase, as would be the case when the variability is due to a rotating, inhomogeneous surface with rotation and orbit synchronized, then it would most efficiently influence the reconstruction of the spectra.

Figure 10 illustrates that the impact on the reconstructed spectra is quite small. In this example, the depth $d_{2}$ of all spectral lines of the secondary component, with the larger velocity amplitude, was modified as follows:

$$
\begin{array}{ll}
x_{2 j}\left(\ln \lambda-v_{2 j} / c\right)=1-\frac{2}{3} d_{2}\left(\ln \lambda-v_{2 j} / c\right) & \text { for } v_{2 j}>\frac{K_{2}}{2} \\
x_{2 j}\left(\ln \lambda-v_{2 j} / c\right)=1-\frac{4}{3} d_{2}\left(\ln \lambda-v_{2 j} / c\right) & \text { for } v_{2 j}<-\frac{K_{2}}{2} \\
x_{2 j}\left(\ln \lambda-v_{2 j} / c\right)=1-d_{2}\left(\ln \lambda-v_{2 j} / c\right) & \text { for }\left|v_{2 j}\right| \leq \frac{K_{2}}{2}
\end{array}
$$

where the $x_{2 j}, j=1 \ldots 16$ are the input spectra $x_{2 j}$ and $d_{2}$ is the deviation from the continuum at $\ln \lambda$ when $v_{2 j}=0$. The lines of 
the secondary are thus twice as deep for the most blue-shifted spectra as compared to the most red-shifted ones, an effect that would not go unnoticed. The reconstructed spectra are biased on the percent level only, with the most rapid change in bias occuring where the line density / line blending is highest.

The bias obtained using the Fourier and the direct algorithm appears at first sight quite different (lower panels of Fig. 10). However, notice that the striking similarity between the bias from the Fourier technique in the middle panel and the difference between the bias and the dot-dashed lines in the lower panel. With the direct method, the impact of the line variability produces a linearly increasing offset that is impeded by the implicit periodicity of the spectra when using the Fourier technique. This confirms the result obtained by Ilijić (2004).

It appears that unnoticed (small) variations in line strength do not seriously prejudice the reconstruction of the (mean) component spectra in the case of a reasonable orbital phase coverage; evidently the fact that the shape of the line profiles is not affected remains an essential requirement.

\section{Elimination of spurious patterns in component spectra}

The purpose of this section is to guide the user with a list of considerations needed to evaluate the reconstructed spectra. When applicable, procedures are described to select physically realistic results from a set of mathematically (quasi)-equivalent solutions. In view of the arguments given in the previous sections, the emphasis lies on low-frequency spurious patterns, since higher-frequency patterns can be avoided by proper observation procedures.

Useful indications about the uniqueness of the solutions and bias in the observed spectra can be retrieved from simple checks on the data and from the computation of condition numbers.

First of all, the total observed line blocking over the considered spectral interval at different orbital phases is an indicator for time-(in)dependent dilution effects in the spectral lines of the components by the combined continuum. When studying a system without light variability, the measured line blocking should be constant within the uncertainties due to the noise in the spectra and, in the case that the selected wavelength interval does not have pure continuum near the edges, the change in the line blocking factor due to Doppler shifts of spectral lines leaving or entering the wavelength range. Often, these uncertainties will turn out to be much smaller than the sensitivity of the measured line blocking to the placement of the continuum. As a consequence, with the hypothesis of a constant-light system, one may obtain a consistent placement of the continuum requiring explicitly a time-independent line blocking. In the more general case of systems with time-dependent relative light ratios and components with different line blocking factors, the line blocking measurements on the observed spectra may indicate that the light variability is or is not correlated with orbital phase. A more detailed inspection may give hints on whether eclipses, a non-spherical shape, intrinsic light or spectral linestrength variations, or simply an incorrect continuum placement is responsible for the changing line blocking.

If the line-blocking measurements, or/and any other knowledge on the system, indicate a significant time-dependent dilution effect, then it is useful to consider how to weigh the different spectra (e.g. in the low Fourier modes, see Sect. 4.1.1) to stabilise the equations as well as possible. The spectra with the most different spectral line dilution, as compared to the majority of the observations, deserve an increased weight on the condition that the changes in line-blocking are not caused by inconsistent data reduction. The arguments for and against such weighting schemes should be carefully considered. In favour of such weighting scheme is the reduction in the level of indeterminacy of (some) equations, and the reduction of the transfer of bias to the component spectra in the case that the spectra with the larger weight are easier to normalise, which is indeed the case for mid-eclipse spectra. Nevertheless, when deciding on the weight to be applied one should take into consideration that the random noise in high-weighted spectra counts heavier in the component spectra (a disadvantage that can be countered by optimising the observation procedure) and that, in the specific case of partial eclipses, the assumption of time-independent line profiles will be invalid to some extent (resulting in a less trustworthy reconstruction of line profiles). The disadvantages are more easily circumvented in the algorithm using the Fourier components of the spectra. Using the velocity-space approach, Harries et al. (2003) did not use any spectra in eclipse because of the deviating line profiles, and they required the line strengths in the spectra of the two components to be in the expected ratio for the derived spectral types to normalise the reconstructed spectra, rather than exploiting the observed change in the dilution of the spectral lines in eclipse.

When the (quasi)-indeterminacy of the low-frequency spectral components is unavoidable, then the number of illconditioned modes and the bias in the input-data/model determine the complexity of the problem. When the wavelength interval is not much longer than the region spanned by the extreme relative Doppler shifts, the mode 0 will be the only one that is ill-conditioned (in absence of bias) and the reconstructed spectra will not be deformed by undulations.

Reconstruction of longer wavelength intervals is advantageous in the absence of numerous continuum windows, but implies a larger risk for spurious low-frequency components in the reconstructed spectra and may require taking the wavelengthdependence of the light factors into account. Bias in the observed spectra or the applied model may affect all frequencies. In both cases, an empirical procedure may be applied that preserves the goodness-of-fit in the comparison to the observed spectra, but reduces the apparently unphysical components. This procedure, described below, exploits external knowledge of the shape of the single-component spectra, especially of the presence of continuum windows and the flatness of the normalised continuum.

From this point on, the reconstructed solution will be referred to as the pseudo-spectra $p_{k}(\ln \lambda)$. The true component spectra $x_{k}(\ln \lambda)$ may differ from the pseudo-spectra,

$p_{k}(\ln \lambda)=x_{k}(\ln \lambda)+\frac{1}{\ell_{k}} z_{k}(\ln \lambda)$

where the spurious pattern $z_{k}(\ln \lambda)$ is a much smoother function than the spectra and the patterns for different components can be related by $\sum_{k} z_{k}(\ln \lambda)=0$ to preserve in very good approximation the goodness-of-fit: the increase in rms scatter will be due only to the Doppler shifting of the $z_{k}(\ln \lambda)$.

In the case of two components, $p_{1,2}=x_{1,2} \pm z / \ell_{1,2}$, the functions

$\pm \ell_{1,2}\left(p_{1,2}(\ln \lambda)-1\right)$

which can be also written in terms of the true spectra $x_{1,2}$ and the spurious pattern $z$ as

$\pm \ell_{1,2}\left(x_{1,2}(\ln \lambda)-1\right)+z(\ln \lambda)$

define a corridor of "continuum" shape and offset $z$ with the absorption lines of $x_{1}$ appearing below the continuum and the 
absorption lines of $x_{2}$ being reversed to emission. Corrections $\mp z / \ell_{1,2}$ may then be applied to the pseudo-spectra.

Any known continuum windows in either component help in defining the local level and the shape of the spurious pattern. In the absence of bias, this shape is fully defined by the ill-determined low-mode Fourier components and will often be nearly sinusoidal. When higher-mode Fourier components are needed, this signals bias in the normalisation of the observed spectra or indicates that the model assumptions applied during the reconstruction of the spectra are not strictly valid.

If, in a higher multiplicity system, there is a pair of stars whose mutual light ratio is the same in all observed spectra, while the ratio of their lights to the remaining light of the system changes, the above procedure can be applied with only a minor technical remark: labeling the stars with 1 and 2, one has $\ell_{1 j} / \ell_{2 j}=r_{12}$, so in expression 25 we set $\ell_{1}=\ell_{1 j}$ and $\ell_{2}=\ell_{2 j}$, with the choice of $j$ being irrelevant. If, on the other hand, more stars have light ratios mutually independent of time, the procedure described above must be applied stepwise: first considering one component $p_{k}$ in relation to the combination of the pseudospectra of all other components together, the functions

$\ell_{k}\left(x_{k}(\ln \lambda)-1\right)+z_{k}(\ln \lambda)$ and $-\sum_{k^{\prime} \neq k} \ell_{k^{\prime}}\left(x_{k^{\prime}}(\ln \lambda)-1\right)+z_{k}(\ln \lambda)$

define a corridor of "continuum" shape and offset $z_{k}$. After application of the additive corrections to all pseudo-spectra, the same procedure may be continued with one of the $K-1$ components $p_{k^{\prime}}$ in relation to the combination of the pseudo-spectra of the $K-2$ remaining ones, and so on. It may be wise to single-out first the fainter constant-light components or those with many bins having a continuum flux.

For a light-ratio varying marginally with time, such that the equations remained ill-conditioned in the lowest modes, the procedure above can be applied using a time-average of the lightratio. If evidence for a wrong normalisation in the observed spectra is detected, the most rigorous approach would be, evidently, to search for improving the normalisation of the observed spectra. In practice, this procedure is more laborious than the empirical correction procedure suggested above. Nevertheless, an example of this rigorous approach is enclosed here because it shows how very obvious undulations can be strongly reduced by very small changes to the continuum level, especially when dealing with relatively-faint components (Fig. 11).

The example concerns the triple system RV Crt (described in Sect. 4.3). The reconstruction of the low-frequency part of the spectrum of the bright component in the close-binary is welldetermined by the inclusion of a spectrum in the totality of the eclipse by the faint companion; the low-frequency part of the other component spectra is ill-determined because their relative light-ratio varies only owing to the slightly non-spherical shape of the fainter close-binary component. The upper panel of Fig. 11 shows the reconstructed spectra. Several of the strong lines are due to $\mathrm{Ca}$ I. The continuum of the bright close-binary component is flat, but the fainter component shows an undulatory continuum, and the distant component shows an opposite low-amplitude undulation in its continuum. Therefore, a sinusoidal correction depending on orbital phase was applied to the observed spectra (Eq. (23)), given by

$\epsilon_{j}(\ln \lambda)=\alpha \sin 2 \pi \phi_{j} \cos 2 \pi \frac{n}{2048}$

where the bin index $n=1, \ldots, 2048$ over the wavelength region, $\phi$ is the orbital phase, and $\alpha$ is the amplitude of the sinusoidal correction applied to the intensities. A correction of this
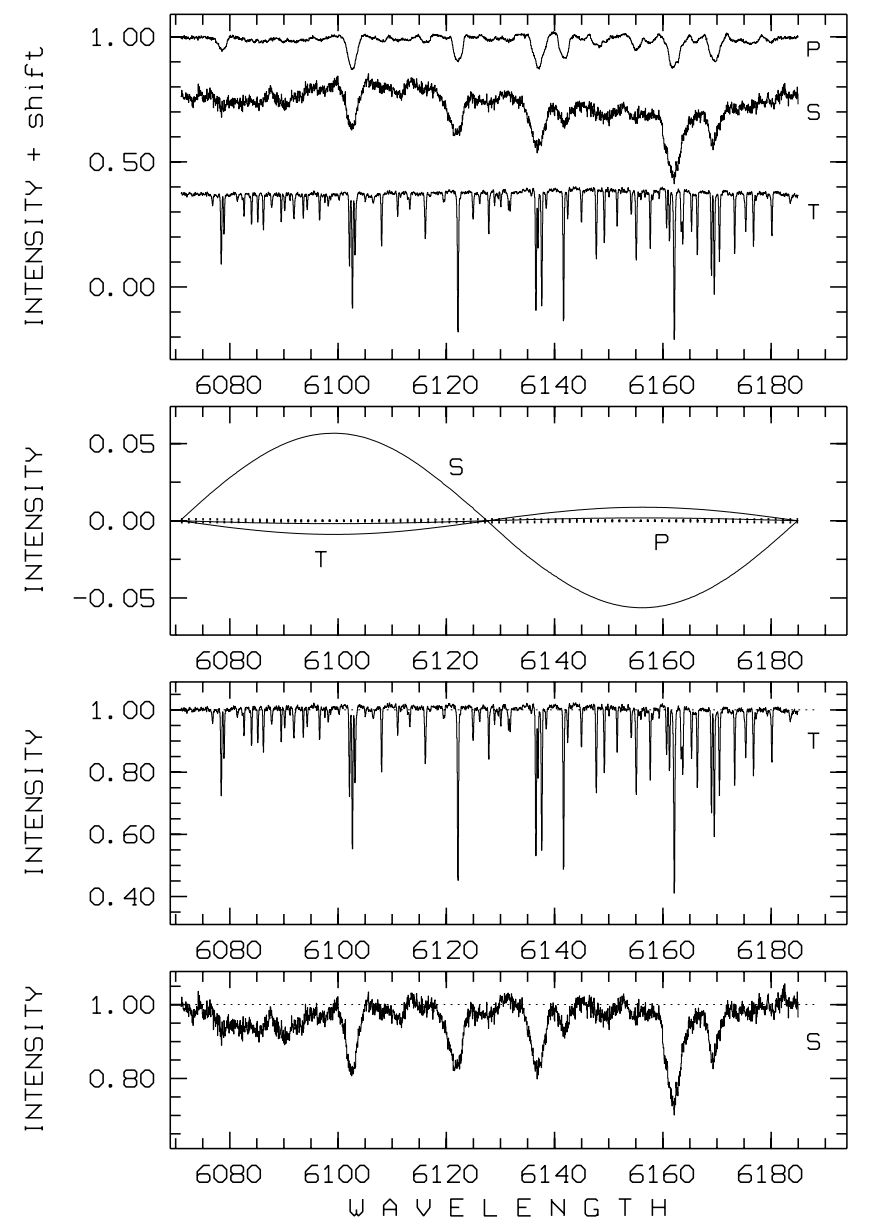

Fig. 11. Reduction of the amplitude of the SVD-induced patterns, superposed on the continuum of reconstructed spectra, by a change in the normalisation of the input spectra. From upper to lower panel: reconstructed spectra of primary $(\mathrm{P})$, secondary $(\mathrm{S})$ and distant companion (T) with the original normalisation, $\mathrm{S}$ and $\mathrm{T}$ are vertically shifted; applied maximum correction to the observed continua (dotted lines, $\sin 2 \pi \phi_{j}= \pm 1$ in Eq. (26)) and difference between the spectra reconstructed starting from the original and the corrected normalisation (labeled full lines); the spectra of $\mathrm{T}$ and $\mathrm{S}$ reconstructed with the improved normalisation.

type assumes that the simpler spectrum in total eclipse was normalised correctly, but that the underlying broader features due to the Doppler-shifted lines of the close-binary component induced a normalisation error. Choosing $\alpha=10^{-3}$, i.e., changing the continuum never by more than $0.1 \%$ (and in several of the $41 \mathrm{spec}-$ tra less) results in satisfactorily flat continua in all reconstructed spectra without enhancing the residuals to the observed spectra. The effect on the reconstructed spectra is seen in the three lower panels of Fig. 11. Figure 12 shows the application of the empirical procedure (determination of $z(\ln \lambda)$ in the case of a pair of stars whose mutual light ratio is approximately constant while their light relative to the third component varies). The chosen $z$ is restricted mostly by the data of the fainter component because the correction to be applied to the reconstructed spectrum is amplified by a larger factor, $\ell_{2} /\left(\ell_{2}+\ell_{3}\right)$. The rectified spectra are very similar, but not identical, to the ones obtained with the rigorous approach.

This example shows that the occurrence of an undulation that deforms strongly the spectrum of a faint component can be triggered by very small errors in the normalisation of the observed spectra. It is not an indication of failure of the method, 

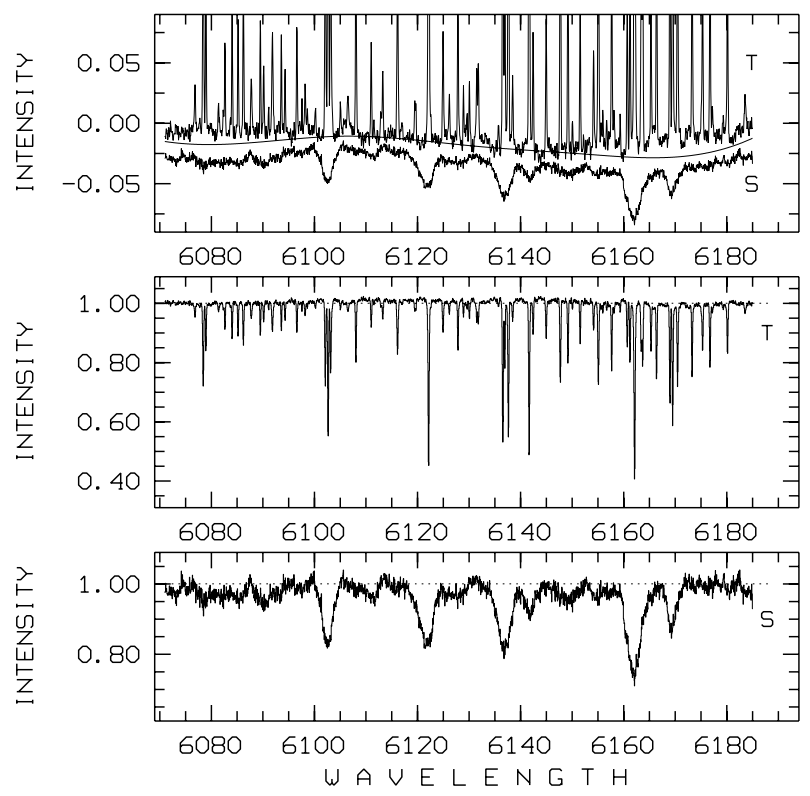

Fig. 12. Reduction of the amplitude of the SVD-induced patterns, superposed on the continuum of reconstructed spectra, by application of the empirical "corridor" procedure. The upper panel shows the functions defining the corridor (for clarity shifted by +0.01 for $T$ and -0.01 for $S$ to move the noise out of the corridor), the lower panels show the reconstructed spectra.

but rather an indicator of how strong the method is: it "sees" that the input data are not consistent and generates a warning about it in the form of a deformation of the reconstructed spectrum. Since the intensity of weak lines is more strongly affected by a misplacement of the continuum, the effect is most obvious in the component spectrum whose lines are less deep in the observed spectrum. This will almost always be the fainter component.

Setting the dilution factor for the spectral lines free, as is done in some applications to deal with spectra in (partial) eclipses (Hadrava 1997, 2004b), will also provide, to some extent, a flatter continuum: a smaller dilution of the spectral lines for the blue-shifted component (and a, relatively, larger dilution of the spectral lines for the red-shifted component) corresponds, very roughly, to putting the continuum higher at shorter wavelengths and lower at longer wavelengths. In a more detailed view, there are of course differences because the lines of both components are distributed over the whole spectral interval, and because a change in continuum level has a greater effect on the strength of the weak lines than the strong ones. This allows us to discern between continuum misplacements and variable line dilution. The statement we make here is that the two types of parameters are not fully independent of eachother.

\section{Conclusions}

The equations governing the separation of component spectra have been analysed with the purpose to discuss under which conditions they lead to well-determined solutions, and how a proper set of composite spectra can be defined. We show that the algorithms acting either on the Fourier components of the spectra or on the spectra in velocity coordinates are equivalent but each one has its own flexibilities to deal (better) with specific requirements related to the binning of the input spectra, the lack of continuum windows at the edges, the identification of the shape of spurious components, etc.
Assuming input spectra with pure random noise, the main conclusions are:

- Whether the equations are well-conditioned depends on the distribution of the input spectra over the Doppler-velocity range covered during the orbit, and on the relative light ratios of the components. The adequacy of the set of input spectra for the purpose of reconstructing the component spectra should be evaluated preferentially during the process of collecting the observations.

- When the equations are ill-conditioned, use of the singularvalue decomposition method is mandatory, also for the algorithm based on the Fourier components of the spectra.

- SVD leads to a mathematically preferable solution, but it should never be considered to be a unique solution from the point of view of the physics. Introduction of external knowledge on the physics of single-star spectra may give preference to a mathematically (quasi-)equivalent solution that differs from the SVD solution. Therefore, it is important to acknowledge when the solution is not unique owing to mathematical reasons and to select all solutions acceptable from the viewpoint of the involved physics.

- With few exceptions (vanishingly faint components, or very specific distributions of the set of input spectra over the orbit), the non-uniqueness of the solutions is restricted to the low-frequency Fourier components of the spectra.

- A necessary condition for the set of equations being wellconditioned is that the dilution of the spectral lines by the continuum of each other component varies significantly over the data-set, i.e. the, ratio of the relative light factors for each couple of components should vary significantly. Hence, spectra in eclipse are particularly useful.

- In absence of a time-variable dilution effect, the zero-point of the component spectra is ill-determined unless the spectral region contains completely black absorption lines, or external information about the depth of some lines in component spectra is available.

- Although spectra obtained in partial eclipse do not fulfill the requirement that the shape of the line profiles of the partially eclipsed component is identical to the shape outside of the eclipse, such spectra are important to extract the physically-correct solution for the low-frequency spectral components. Mid-eclipse spectra are especially useful. The algorithm based on the Fourier components of the spectra allows us to use the spectra in partial eclipse for the lowest frequencies, while neglecting them for all other frequencies. The use of mid-eclipse spectra is beneficial for $m=0$ and, if the wavelength interval is large in units of Doppler shifts and line widths, for a few more modes for which the condition number $C_{m}$ computed with the spectra in partial eclipse included is significantly lower than without them (say at least a factor of two). The specific cases mentioned in Fig. 6 show that the upper limit for $m$ lies commonly in the range 1-5.

The normalised input spectra, obtained after a complex data reduction process, are likely not free from systematic errors. The progression of the bias in the case of (normalisation) errors correlating with the orbital phase was discussed, since it is the worst possible scenario. The main conclusions are:

- A large amplification of any bias that correlates with orbital phase is observed in the solution process in absence of timevariable light ratios between couples of components. The bias is transferred primarily to the fainter component(s) and 
its amplitude increases linearly with the length of the reconstructed spectral interval in units of the sum of the Doppler shifts for each couple of components.

- The two types of algorithms discussed in this paper react in a similar, but not necessarily identical way on bias in the input data, because of the implicit requirement for periodic data in the Fourier algorithm.

- In first order, an orbital-phase correlated low-frequency bias in the normalisation of the input spectra, or (neglected) orbital-phase correlated changes in the line strengths of a component may produce the same kind of bias in the reconstructed spectra.

Finally, we described in Sect. 6 practical approaches to remove or reduce the spurious patterns introduced either by illconditioned equations or by observational bias. We also discussed the laborious procedure of improving the normalisation of the observed spectra and iterating the reconstruction procedure, as an a-posteriori correction procedure for removal of spurious patterns from the reconstructed spectra. The need for coupled corrections in the reconstructed spectra is emphasized, guaranteeing that the observed line blocking is preserved in the combination of the reconstructed component spectra.

In conclusion, the reconstruction algorithms are most powerful when applied to eclipsing binaries because the timevariable dilution effect on the spectral lines fully determines the strength of the lines in the normalised component spectra. A flat normalised continuum and the correct zero-intensity level are reconstructed in a natural way when the weight of the observations is distributed well over the range in line-strength dilution and velocity amplitudes. Caution is required only with lines extending to within $K_{1}+K_{2}$ of the edges of the spectrum, and possibly over a twice larger region near the edges when using wrap-around algorithms. Except near the edges, a full astrophysical analysis of the reconstructed component spectrum is as straightforward as for an observed single-star spectrum. The global characteristics of the atmosphere and its chemical composition can be derived with precision.

In most other situations, as shown in Sect. 2, external information is necessary to determine the zero-intensity level unambiguously, and the shape of broader spectral lines may appear distorted by low-frequency, spurious patterns. For studies of sharp lines, the local continuum is easy to estimate and any uncertainty in continuum level has much less influence on the interpretation of the strength and the exact shape of the line than in the case of a broad-lined spectrum. The distortions discussed in this paper are then of minor practical importance. However, for fainter components, with spurious patterns reaching higher amplitude, and for fast rotators (where the location of the continuum has larger impact) and intrinsically very broad lines (as the hydrogen lines in hotter stars or the Ca II lines in cooler stars) the distortion of the continuum is more likely to have a dominant impact on the quality of the analysis, and it is beneficial to consider the approaches described in this paper. An early application is described in Pavlovski \& Hensberge (2005), showing that the abundances of the main chemical species in an early-B type binary can be derived with a precision better than 0.1 dex at rotation velocities around $100 \mathrm{~km} \mathrm{~s}^{-1}$. In our opinion, the fact that the low-frequency components of reconstructed spectra are not always determined uniquely has been misinterpreted frequently as a basic obstacle to reconstruct large stretches of spectrum and as a barrier lowering the confidence in the algorithm. The fact that the patterns imposed on different components are strictly interrelated provides a very helpful constraint for recovering the true low-frequency contributions to the component spectra, but it is often not yet exploited. Consistency requirements on the physics derived from different spectral lines, as mentioned in Sect. 2, also constrain otherwise unknown light ratios. Future applications of the technique on a variety of systems will undoubtedly show that significantly more information can be derived from reconstructed spectra than obtained at present. Abundance analyses, detailed studies of line-shape variations in pulsating stars belonging to spectroscopically multiple systems (Uytterhoeven et al. 2005), co-existence of spectroscopically peculiar and normal components in multiple systems (Frémat et al. 2005), and their detailed analysis over a large wavelength range are realistic aims.

Within the broader context of the disentangling of multiple spectra, involving the determination of the orbital parameters, future efforts should address the bias introduced possibly in the reconstructed spectra by errors (bias) in the orbital parameters. This type of error progression is not included in the present discussion. Intuitively, such errors are expected to alter somewhat the shape of the spectral features rather than their strength, probably by a combination of some level of spurious broadening and asymmetry. In contrast to the sources of bias discussed in this paper, which distort the low-frequency modes in the component spectra, wrong orbital parameters may affect higher-frequency modes. However, disentangling methods are capable of using velocity information at orbital phases not accessible with classical methods and do not depend on templates, such that the errors on the Doppler velocities may be expected to remain small for well-determined data sets. Nevertheless, a quantitative analysis would contribute to a correct estimation of the uncertainties on astrophysical parameters derived from reconstructed spectra.

Acknowledgements. H.H. thanks L. P. R. Vaz for the hospitality and financial support received during several visits to UFMG, Brazil in the period 2002-2006 (FAPEMIG grants CEX-0080-01 and CEX-0058-04 and CAPES. through the resources of the Graduate Physics Course of UFMG). H.H. and K.B.V.T. thank K. Pavlovski and SI for the hospitality, use of infrastructure, and financial support received during stays at the University of Zagreb (and once at the University of Vienna), and acknowledge support of the "IAP P5/36" project financed by the Belgian Science Policy. K.B.V.T. gratefully acknowledges support received from the Brazilian agencies CNPq, CAPES and FAPEMIG, and, especially, the hospitality received at Royal Observatory of Belgium from March 2006 to April 2007. The authors thank G. Torres and V. Bakis for making available observational data and a draft of a paper, respectively, and L. P. R. Vaz for supporting computations of light factors from photometric data and also, together with K. Pavlovski, for the critical reading of the paper. This work used extensively the software codes KOREL (made publicly available by P. Hadrava), FDBINARY and CRES (both made publicly available by SI) for the disentangling of spectra, and the ESOMIDAS software for astronomical data reduction and analysis.

\section{Appendix A: Analytical solutions for a very simple data-set}

In order to gain insight into the structure of the equations, their possible degeneracies and the origin of spurious patterns, the solution for a very simple data-set is presented explicitly in an analytical form, both in velocity space and in Fourier space.

Consider a binary system with component spectra

$x_{k i}=\left(\begin{array}{cccccc}\cdots & 1 & 1 & 2 / 3 & 1 & \cdots \\ \cdots & 1 & 1 / 3 & 1 & 1 & \cdots\end{array}\right)$

and constant light factors $\ell_{1}=3 / 4$ and $\ell_{2}=1 / 4$. The radial velocities (in pixel units) for the three orbital phases are:

$\beta_{k j}=\left(\begin{array}{lll}-2 & 0 & +2 \\ +1 & 0 & -1\end{array}\right)$ 
The observed composite spectra truncated to eight data bins are:

$y_{j i}=\left(\begin{array}{cccccccc}3 / 4 & 1 & 5 / 6 & 1 & 1 & 1 & 1 & 1 \\ 1 & 5 / 6 & 3 / 4 & 1 & 1 & 1 & 1 & 1 \\ 5 / 6 & 1 & 1 & 1 & 3 / 4 & 1 & 1 & 1\end{array}\right)$.

It is irrelevant at which edge(s) the three bins with intensities at the continuum level are added.

\section{A.1. Velocity-space algorithm}

Considering $\sigma_{j i}=1$, the equations for the velocity-space algorithm are:

$\left(\begin{array}{c}x_{1,1} \\ x_{1,2} \\ x_{1,3} \\ x_{1,4} \\ x_{1,5} \\ x_{1,6} \\ x_{1,7} \\ x_{1,8} \\ x_{1,9} \\ x_{1,10} \\ x_{1,11} \\ x_{1,12} \\ x_{2,1} \\ x_{2,2} \\ x_{2,3} \\ x_{2,4} \\ x_{2,5} \\ x_{2,6} \\ x_{2,7} \\ x_{2,8} \\ x_{2,9} \\ x_{2,10}\end{array}\right)=\left(\begin{array}{c}3 / 4 \\ 1 \\ 5 / 6 \\ 1 \\ 1 \\ 1 \\ 1 \\ 1 \\ 1 \\ 5 / 6 \\ 3 / 4 \\ 1 \\ 1 \\ 1 \\ 1 \\ 1 \\ 5 / 6 \\ 1 \\ 1 \\ 1 \\ 3 / 4 \\ 1 \\ 1 \\ 1\end{array}\right)=$

where the design matrix $\boldsymbol{A}$ reads

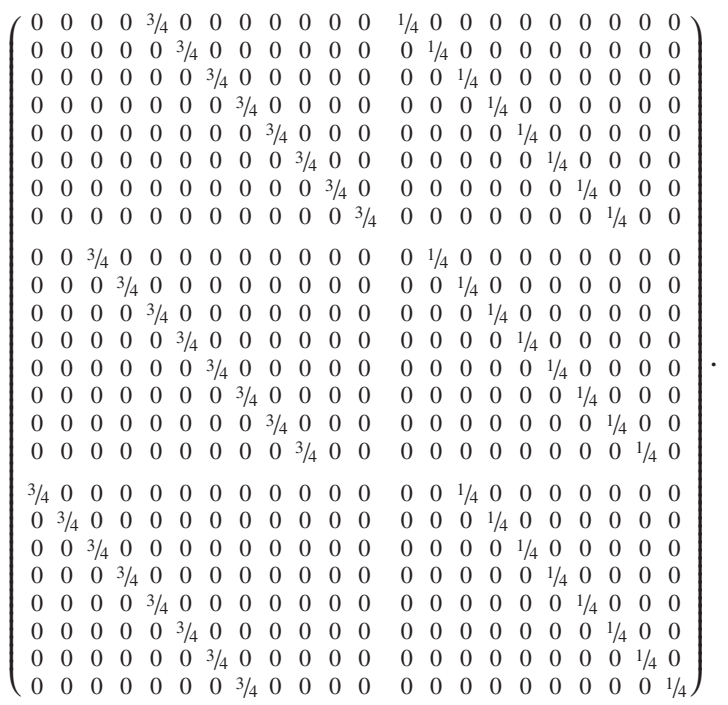

The matrix $\boldsymbol{A}$ holds the information about the $\beta_{k j}$. The diagonal in the sub-blocks changes to the right and to the left in accordance with the Doppler shifts (Eq. (A.2)). Note that the size of the design matrix depends on the extreme Doppler shifts: 12 columns $(2+8+2)$ are used to track the information related to the first component and 10 columns $(1+8+1)$ for the second one. Writing Eq. (A.4) explicitly:

$$
\begin{aligned}
\frac{3}{4} x_{1, i}+\frac{1}{4} x_{2, i-4} & =y_{1, i-4} & & (i=5, \ldots, 12) \\
\frac{3}{4} x_{1, i}+\frac{1}{4} x_{2, i-1} & =y_{2, i-2} & & (i=3, \ldots, 10) \\
\frac{3}{4} x_{1, i}+\frac{1}{4} x_{2, i+2} & =y_{3, i} & & (i=1, \ldots, 8)
\end{aligned}
$$

leads to 24 coupled equations. The solution is found minimizing $\chi^{2}=|\boldsymbol{b}-\boldsymbol{A} \cdot \boldsymbol{x}|^{2}$. The resulting 22 normal equations are:

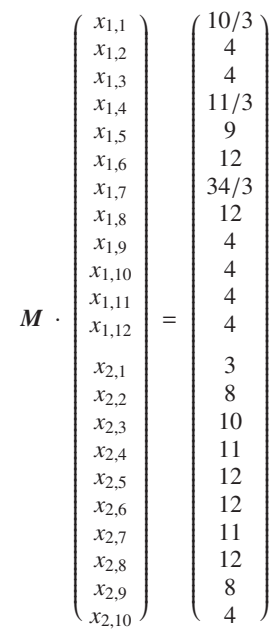

where the normal matrix $\boldsymbol{M}=\boldsymbol{A}^{\dagger} \boldsymbol{A}$ is a square matrix with dimension 22 and reads:

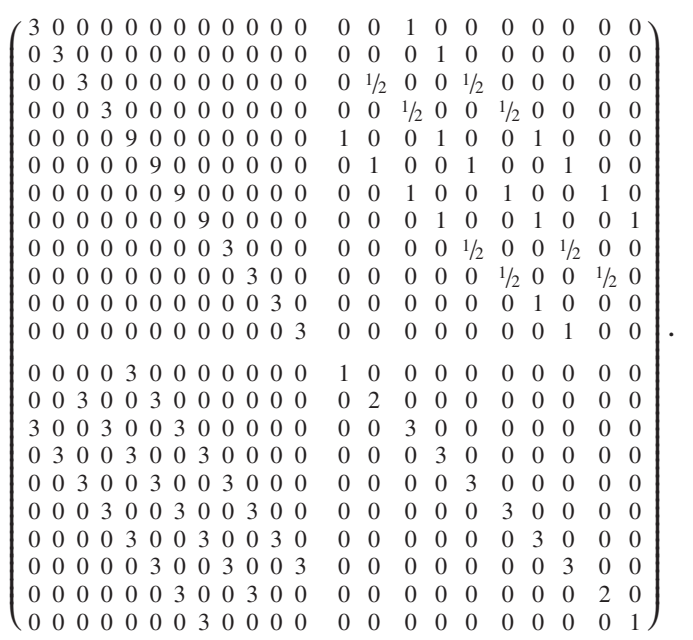

Consecutive rows correspond to the partial derivatives to the consecutive components of the vectors $x_{1}$ and $x_{2}$, respectively, in Eq. (A.4). Grouping the equations (rows of the matrix and the vectors) such that those containing the vector elements $x_{1 j}$ and $x_{2 k}$, with $j$ and $k$ progressing by multiples of 3 , are in subsequent rows (see the order of the elements in the $x$-vector in the equation below) and then re-arranging the columns of the matrix, to obtain non-zero sub-matrices around the diagonal leads to:

$\left(\begin{array}{ccc}\boldsymbol{M}_{a} & \mathbf{0} & \mathbf{0} \\ \mathbf{0} & \boldsymbol{M}_{b} & \mathbf{0} \\ \mathbf{0} & \mathbf{0} & \boldsymbol{M}_{c}\end{array}\right) \cdot\left(\begin{array}{c}x_{1,1} \\ x_{1,4} \\ x_{1,7} \\ x_{1,10} \\ x_{2,3} \\ x_{2,6} \\ x_{2,9} \\ x_{1,2} \\ x_{1,5} \\ x_{1,8} \\ x_{1,11} \\ x_{2,1} \\ x_{2,4} \\ x_{2,7} \\ x_{2,10} \\ x_{1,3} \\ x_{1,6} \\ x_{1,9} \\ x_{1,12} \\ x_{2,2} \\ x_{2,5} \\ x_{2,8}\end{array}\right)=\left(\begin{array}{c}10 / 3 \\ 11 / 3 \\ 34 / 3 \\ 4 \\ 10 \\ 12 \\ 8 \\ 4 \\ 9 \\ 12 \\ 4 \\ 3 \\ 11 \\ 11 \\ 4 \\ 4 \\ 12 \\ 4 \\ 4 \\ 8 \\ 12 \\ 12\end{array}\right)$ 
where $\mathbf{0}$ are null matrices with dimensions conform to the subblocks $\boldsymbol{M}_{a}, \boldsymbol{M}_{b}$ and $\boldsymbol{M}_{c}$ and

$\boldsymbol{M}_{a}=\left(\begin{array}{ccccccc}3 & 0 & 0 & 0 & 1 & 0 & 0 \\ 0 & 3 & 0 & 0 & 1 / 2 & 1 / 2 & 0 \\ 0 & 0 & 9 & 0 & 1 & 1 & 1 \\ 0 & 0 & 0 & 3 & 0 & 1 / 2 & 1 / 2 \\ 3 & 3 & 3 & 0 & 3 & 0 & 0 \\ 0 & 3 & 3 & 3 & 0 & 3 & 0 \\ 0 & 0 & 3 & 3 & 0 & 0 & 2\end{array}\right)$

$\boldsymbol{M}_{b}=\left(\begin{array}{llllllll}3 & 0 & 0 & 0 & 0 & 1 & 0 & 0 \\ 0 & 9 & 0 & 0 & 1 & 1 & 1 & 0 \\ 0 & 0 & 9 & 0 & 0 & 1 & 1 & 1 \\ 0 & 0 & 0 & 3 & 0 & 0 & 1 & 0 \\ 0 & 3 & 0 & 0 & 1 & 0 & 0 & 0 \\ 3 & 3 & 3 & 0 & 0 & 3 & 0 & 0 \\ 0 & 3 & 3 & 3 & 0 & 0 & 3 & 0 \\ 0 & 0 & 3 & 0 & 0 & 0 & 0 & 1\end{array}\right)$

$\boldsymbol{M}_{c}=\left(\begin{array}{ccccccc}3 & 0 & 0 & 0 & 1 / 2 & 1 / 2 & 0 \\ 0 & 9 & 0 & 0 & 1 & 1 & 1 \\ 0 & 0 & 3 & 0 & 0 & 1 / 2 & 1 / 2 \\ 0 & 0 & 0 & 3 & 0 & 0 & 1 \\ 3 & 3 & 0 & 0 & 2 & 0 & 0 \\ 3 & 3 & 3 & 0 & 0 & 3 & 0 \\ 0 & 3 & 3 & 3 & 0 & 0 & 3\end{array}\right)$.

The problem is now separated in 3 subsystems with independent sets of variables. The determinant of each subset is zero. Hence, there is a triple infinity of solutions:

$$
\begin{aligned}
x_{1,1} & =x_{1,4}=x_{1,7}=x_{1,10}=\frac{4-x_{2,6}}{3}=\frac{10-3 x_{2,3}}{9}=\frac{4-x_{2,9}}{3} \\
x_{1,2} & =x_{1,5}+\frac{1}{3}=x_{1,8} \\
& =\frac{4-x_{2,1}}{3}=\frac{4-x_{2,4}}{3}=\frac{4-x_{2,7}}{3}=\frac{4-x_{2,10}}{3} \\
x_{1,3} & =x_{1,6}=x_{1,9}=x_{1,12}=\frac{4-x_{2,2}}{3}=\frac{4-x_{2,5}}{3}=\frac{4-x_{2,8}}{3} .
\end{aligned}
$$

The physically-correct solution is recovered for $x_{11}=x_{12}=$ $x_{13}=1$. Any other choice for these variables results in a superimposed pattern on the reconstructed component spectra, with a "periodicity" of 3 pixels. Applying the SVD on the design matrix (Eq. (A.4)) leads to the solution shown in Table A.1. It is not the physically-correct one, but the one that minimizes the length of the solution vector in each subset, i.e.: $\sum_{j=0}^{3} x_{1,3 j+1}^{2}+\sum_{j=1}^{3} x_{2,3 j}^{2}$, $\sum_{j=0}^{3} x_{1,3 j+2}^{2}+\sum_{j=0}^{3} x_{2,3 j+1}^{2}, \sum_{j=1}^{4} x_{1,3 j}^{2}+\sum_{j=0}^{3} x_{2,3 j+2}^{2}$. The minimum lengths found after substitution of (A.13) into these relations impose $x_{1,1}=\frac{34}{31}, x_{1,2}=\frac{29}{24}$, and $x_{1,3}=\frac{36}{31}$. The periodicity of 3 pixels in the spurious component corresponds to all $\left|\left(\beta_{2 j}-\beta_{1 j}\right)-\left(\beta_{2 j^{\prime}}-\beta_{1 j^{\prime}}\right)\right|$ being multiples of 3 .

The SVD solution constrains the solution well in a mathematical sense, but cannot reconstruct the true component spectra when the equations are degenerated. The shape of the spurious pattern depends both on the different size of the block matrices $\boldsymbol{M}_{a}, \boldsymbol{M}_{b}$, and $\boldsymbol{M}_{c}$, and on the shape of the observed spectra. It is easily checked that a pattern would be present even if the observed spectra were pure continuum (though in this case, two of three subsequent points would have identical values since $\boldsymbol{M}_{a}$ and $\boldsymbol{M}_{c}$ have the same size).

\section{A.2. Fourier-space algorithm}

Considering $\sigma_{j, m}=1$, the equations to solve order $m$ with the Fourier-space algorithm are:

$$
\left(\begin{array}{ll}
\left(\boldsymbol{F}_{m}\right)_{11} & \left(\boldsymbol{F}_{m}\right)_{12} \\
\left(\boldsymbol{F}_{m}\right)_{21} & \left(\boldsymbol{F}_{m}\right)_{22} \\
\left(\boldsymbol{F}_{m}\right)_{31} & \left(\boldsymbol{F}_{m}\right)_{32}
\end{array}\right)\left(\begin{array}{l}
\tilde{x}_{1, m} \\
\tilde{x}_{2, m}
\end{array}\right)=\left(\begin{array}{l}
\tilde{y}_{1, m} \\
\tilde{y}_{2, m} \\
\tilde{y}_{3, m}
\end{array}\right)
$$

Table A.1. The reconstructed spectra of the components in velocity and Fourier space. The SVD-induced patterns in velocity space are in boldface. Note that the amplitude of the spurious component superposed on $x_{1}$ is exactly three times larger than for $x_{2}$ and in the opposite sense. This reflects the light ratio between the two components.

\begin{tabular}{ll|cc}
\hline \hline \multicolumn{2}{c|}{ Velocity-space } & \multicolumn{2}{c}{ Fourier-space } \\
$x_{1}$ & $x_{2}$ & $x_{1}$ & $x_{2}$ \\
\hline $1+\mathbf{3} / \mathbf{3 1}$ & - & - & - \\
$1+\mathbf{5} / \mathbf{2 4}$ & $1-\mathbf{5} / \mathbf{8}$ & - & - \\
$1+\mathbf{5} / \mathbf{3 1}$ & $1-\mathbf{1 5} / \mathbf{3 1}$ & 1 & 1 \\
$1+\mathbf{3} / \mathbf{3 1}$ & $1 / 3-\mathbf{9} / \mathbf{3 1}$ & 1 & $1 / 3$ \\
$2 / 3+\mathbf{5} / \mathbf{2 4}$ & $1-\mathbf{5} / \mathbf{8}$ & $2 / 3$ & 1 \\
$1+\mathbf{5} / \mathbf{3 1}$ & $1-\mathbf{1 5} / \mathbf{3 1}$ & 1 & 1 \\
$1+\mathbf{3} / \mathbf{3 1}$ & $1-\mathbf{9} / \mathbf{3 1}$ & 1 & 1 \\
$1+\mathbf{5} / \mathbf{2 4}$ & $1-\mathbf{5} / \mathbf{8}$ & 1 & 1 \\
$1+\mathbf{5} / \mathbf{3 1}$ & $1-\mathbf{1 5} / \mathbf{3 1}$ & 1 & 1 \\
$1+\mathbf{3} / \mathbf{3 1}$ & $1-\mathbf{9} / \mathbf{3 1}$ & 1 & 1 \\
$1+\mathbf{5} / \mathbf{2 4}$ & $1-\mathbf{5} / \mathbf{8}$ & - & - \\
$1+\mathbf{5} / \mathbf{3 1}$ & - & - & - \\
\hline
\end{tabular}

Table A.2. The spurious components in the reconstructed spectra when 6 instead of 8 pixels are used. The lay-out of the table is the same as in Table A.1.

\begin{tabular}{ll|ll}
\hline \hline \multicolumn{2}{c|}{ Velocity-space } & \multicolumn{2}{c}{ Fourier-space } \\
$x_{1}$ & $x_{2}$ & $x_{1}$ & $x_{2}$ \\
\hline $1+\mathbf{0}$ & - & - & - \\
$1+\mathbf{1 9} / \mathbf{9 0}$ & $1-\mathbf{1 9 / 3 0}$ & - & - \\
$1+\mathbf{1} / \mathbf{5}$ & $1-\mathbf{3} / \mathbf{5}$ & $1+\mathbf{1 / 5}$ & $1-\mathbf{3} / \mathbf{5}$ \\
$1+\mathbf{0}$ & $1 / 3-\mathbf{0}$ & $1+\mathbf{1} / \mathbf{1 0}$ & $1 / 3-\mathbf{3} / \mathbf{1 0}$ \\
$2 / 3+\mathbf{1 9} / \mathbf{9 0}$ & $1-\mathbf{1 9 / 3 0}$ & $2 / 3+\mathbf{1 3} / \mathbf{6 0}$ & $1-\mathbf{1 3 / 2 0}$ \\
$1+\mathbf{1} / \mathbf{5}$ & $1-\mathbf{3 / 5}$ & $1+\mathbf{1 / 5}$ & $1-\mathbf{3} / \mathbf{5}$ \\
$1+\mathbf{0}$ & $1-\mathbf{0}$ & $1+\mathbf{1} / \mathbf{1 0}$ & $1-\mathbf{3} / \mathbf{1 0}$ \\
$1+\mathbf{1 9} / \mathbf{9 0}$ & $1-\mathbf{1 9} / \mathbf{3 0}$ & $1+\mathbf{1 3} / \mathbf{6 0}$ & $1-\mathbf{1 3} / \mathbf{2 0}$ \\
$1+\mathbf{1} / \mathbf{5}$ & $1-\mathbf{3 / 5}$ & - & - \\
$1+\mathbf{0}$ & - & - & - \\
\hline
\end{tabular}

where, $\left(\boldsymbol{F}_{m}\right)_{j k} \equiv F_{j k, m}=\ell_{k} \mathrm{e}^{-2 \pi \mathrm{i} \frac{m}{8} \beta_{k, j}} ; x_{k, m}$ and $\tilde{y}_{j, m}$ are defined in Eq. (5) (here the index $j$ in $\ell_{k}$ is omitted because the lightfactors are constant in time). The normal equations for mode $m$ then read:

$$
\left(\begin{array}{ll}
\left(\boldsymbol{F}_{m}^{\dagger} \boldsymbol{F}_{m}\right)_{11} & \left(\boldsymbol{F}_{m}^{\dagger} \boldsymbol{F}_{m}\right)_{12} \\
\left(\boldsymbol{F}_{m}^{\dagger} \boldsymbol{F}_{m}\right)_{21} & \left(\boldsymbol{F}_{m}^{\dagger} \boldsymbol{F}_{m}\right)_{22}
\end{array}\right)\left(\begin{array}{c}
\tilde{x}_{1, m} \\
\tilde{x}_{2, m}
\end{array}\right)=\left(\begin{array}{c}
\tilde{Y}_{m}(1) \\
\tilde{Y}_{m}(2)
\end{array}\right)
$$

with the Hermitian matrix:

$\left(\boldsymbol{F}_{m}^{\dagger} \boldsymbol{F}_{m}\right)_{k k^{\prime}}=\sum_{j=1}^{3} \ell_{k} \ell_{k^{\prime}} \mathrm{e}^{2 \pi \mathrm{i} \frac{m}{8}\left(\beta_{k j}-\beta_{k^{\prime} j}\right)}$

and the vector:

$\tilde{Y}_{m}(k)=\sum_{j=1}^{3} \ell_{k} \tilde{y}_{j, m} \mathrm{e}^{2 \pi \mathrm{i} \frac{m}{8} \beta_{k j}}$

The solution of Eq. (A.15) for $m=1,2,3$ and 4 is documented in Table A.3. The inverse Fourier transform of $\tilde{x}_{1, m}$ and $\tilde{x}_{2, m}$ leads, in this particular case, to the exact component spectra $x_{1}$ and $x_{2}$ (see Table A.1). Alternatively, applying SVD directly on the design matrix (Eq. (A.14)), the same result is found.

\section{A.3. Discussion: Fourier versus velocity space}

Why show the reconstructed spectra, in this particular case, a spurious pattern when reconstructed with one algorithm, and not 
Table A.3. Relevant numerical quantities and solution in Fourier-space.

\begin{tabular}{lllll}
\hline \hline & $m=1$ & $m=2$ & $m=3$ & $m=4$ \\
\hline$\left(\boldsymbol{F}_{m}^{\dagger} \boldsymbol{F}_{m}\right)_{11}$ & $\frac{27}{16}$ & $\frac{27}{16}$ & $\frac{27}{16}$ & $\frac{27}{16}$ \\
$\left(\boldsymbol{F}_{m}^{\dagger} \boldsymbol{F}_{m}\right)_{12}$ & $\frac{3}{16}(1-\sqrt{2})$ & $\frac{3}{16}$ & $\frac{3}{16}(1+\sqrt{2})$ & $-\frac{3}{16}$ \\
$\left(\boldsymbol{F}_{m}^{\dagger} \boldsymbol{F}_{m}\right)_{21}$ & $\frac{3}{16}(1-\sqrt{2})$ & $\frac{3}{16}$ & $\frac{3}{16}(1+\sqrt{2})$ & $-\frac{3}{16}$ \\
$\left(\boldsymbol{F}_{m}^{\dagger} \boldsymbol{F}_{m}\right)_{22}$ & $\frac{3}{16}$ & $\frac{3}{16}$ & $\frac{3}{16}$ & $\frac{3}{16}$ \\
$\operatorname{Re}\left(\tilde{Y}_{m}(1)\right)$ & $\frac{3}{4} \frac{2-\sqrt{2}}{96}$ & $\frac{3}{4} \frac{9}{96}$ & $\frac{3}{4} \frac{2+\sqrt{2}}{96}$ & $-\frac{3}{4} \frac{11}{96}$ \\
$\operatorname{Im}\left(\tilde{Y}_{m}(1)\right)$ & $\frac{3}{4} \frac{7+\sqrt{2}}{96}$ & $\frac{3}{4} \frac{2}{96}$ & $\frac{3}{4} \frac{\sqrt{2}-7}{96}$ & 0 \\
$\operatorname{Re}\left(\tilde{Y}_{m}(2)\right)$ & $-\frac{1}{4} \frac{3 \sqrt{2}}{96}$ & $\frac{1}{4} \frac{3}{96}$ & $\frac{1}{4} \frac{3 \sqrt{2}}{96}$ & $\frac{1}{4} \frac{9}{96}$ \\
$\operatorname{Im}\left(\tilde{Y}_{m}(2)\right)$ & $\frac{1}{4} \frac{3}{96}$ & $\frac{1}{4} \frac{6}{96}$ & $-\frac{1}{4} \frac{3}{96}$ & 0 \\
$\tilde{x}_{1, m}$ & $\frac{i}{24}$ & $\frac{1}{24}$ & $-\frac{i}{24}$ & $-\frac{1}{24}$ \\
$\tilde{x}_{2, m}$ & $\frac{\sqrt{2}}{24}(i-1)$ & $\frac{i}{12}$ & $\frac{\sqrt{2}}{24}(i+1)$ & $\frac{1}{12}$ \\
\hline
\end{tabular}

when reconstructed with the other algorithm? The answer lies in the periodicity of the pattern imposed by the velocity differences (3 pixels) and the periodicity of the reconstructed spectra imposed by the length of the spectral interval in the Fourier space ( 8 pixel). The latter implies that the reconstructed spectra have equal intensity in pixels $p$ and $p+8$, while the former imposes equal continuum intensities in pixels $p$ and $p+3$. Both together are only fulfilled for $x_{1,1}=x_{1,2}=x_{1,3}$ such that the difference between the reconstructed spectra and the true component spectra is at most an offset.

It is easy to check that our data analysed over an observed interval of 6 pixels (or any other multiple of 3) also produce a spurious pattern when reconstructing the component spectra with the Fourier space algorithm (Table A.2), or that a case with all $\left|\left(\beta_{2 j}-\beta_{1 j}\right)-\left(\beta_{2 j^{\prime}}-\beta_{1 j^{\prime}}\right)\right|$ multiples of 4 also produces a spurious pattern with both algorithms using interval lengths of $2^{n}$.

\section{A.4. Discussion: amplitude of spurious patterns}

The case worked out explicitly in this appendix is "special" in the sense that it concerns few spectra in a spectral interval not much larger than the Doppler shifts. Hence, the question arises whether differences in the reconstruction may be as large as shown here in cases with more spectra and larger spectral intervals.

First, it is evident that the separation of the design matrix in independent sub-blocks is much less likely when the algorithm is capable of dealing with sub-pixel shifts. We have worked out such examples with few spectra and few pixels (not reproduced explicitly here) and found no spurious patterns.

Secondly, if the spectra are very regularly distributed with regard to the Doppler shifts, but the length of the spectral interval increases, then it can be shown that the amplitude of the spurious pattern tends to zero.

As an example, consider three pure continuum input spectra with the velocity structure defined by (A.2) and a wavelength interval with length $N$ pixels, such that the design matrix will have $2 N+6$ columns and $3 N$ rows. For $N=3 N_{\mathrm{p}}$ the SVD solution leads then to

$$
\begin{aligned}
& x_{1,1}=\frac{\ell_{1}}{\ell_{1}^{2}+\ell_{2}^{2}+\frac{2 \ell_{2}^{2}}{N_{\mathrm{p}}}} \neq x_{1,2}=x_{1,3}=\frac{\ell_{1}}{\ell_{1}^{2}+\ell_{2}^{2}} \\
& x_{2,1}=x_{2,2}=\frac{\ell_{2}}{\ell_{1}^{2}+\ell_{2}^{2}} \neq x_{2,3}=\frac{\ell_{2}}{\ell_{1}^{2}+\ell_{2}^{2}-\frac{2 \ell_{1}^{2}}{2+N_{\mathrm{p}}}},
\end{aligned}
$$

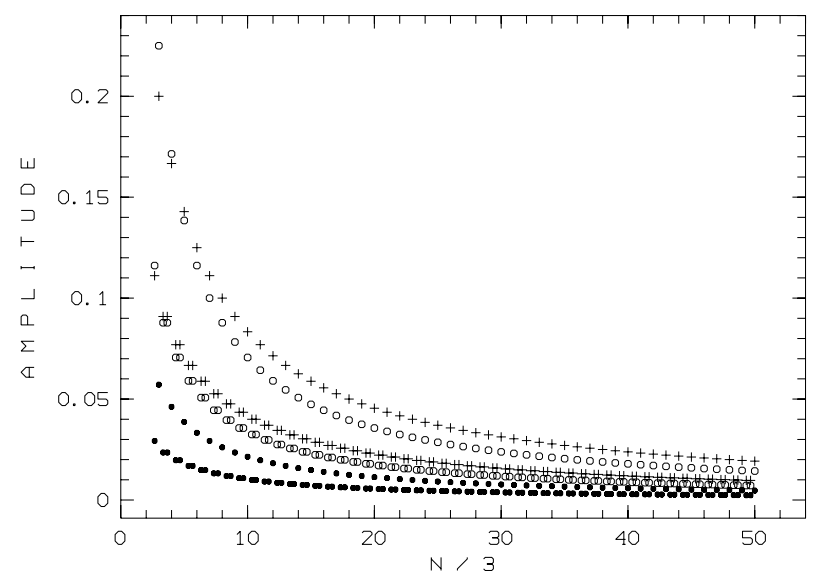

Fig. A.1. Reduction of the amplitude of the SVD-induced patterns, superposed on the continuum of reconstructed spectra, with increasing spectral interval (in units of the periodicity in the pattern) for two cases: $\ell_{1}=\ell_{2}=0.5(+)$ and $\ell_{1}=0.75, \ell_{2}=0.25$ (filled symbols for the bright component, open circles for the faint component). In this particular case, the spurious pattern is more pronounced when $N$ is a multiple of the periodicity.

for $N=3 N_{\mathrm{p}}-1$

$x_{1,1}=x_{1,3}=\frac{\ell_{1}}{\ell_{1}^{2}+\ell_{2}^{2}+\frac{\ell_{2}^{2}}{N_{\mathrm{p}}}} \neq x_{1,2}=\frac{\ell_{1}}{\ell_{1}^{2}+\ell_{2}^{2}}$

$x_{2,1}=\frac{\ell_{2}}{\ell_{1}^{2}+\ell_{2}^{2}} \neq x_{2,2}=x_{2,3}=\frac{\ell_{2}}{\ell_{1}^{2}+\ell_{2}^{2}-\frac{\ell_{1}^{2}}{1+N_{\mathrm{p}}}}$,

for $N=3 N_{\mathrm{p}}-2$

$x_{1,1}=x_{1,2}=\frac{\ell_{1}}{\ell_{1}^{2}+\ell_{2}^{2}+\frac{\ell_{2}^{2}}{N_{\mathrm{p}}}} \neq x_{1,3}=\frac{\ell_{1}}{\ell_{1}^{2}+\ell_{2}^{2}}$
$x_{2,1}=x_{2,3}=\frac{\ell_{2}}{\ell_{1}^{2}+\ell_{2}^{2}-\frac{\ell_{1}^{2}}{1+N_{\mathrm{p}}}} \neq x_{2,2}=\frac{\ell_{2}}{\ell_{1}^{2}+\ell_{2}^{2}}$,

where $N_{\mathrm{p}}$ is the largest integer equal to or smaller than $\frac{N}{3}$, and in all cases the remaining components of the vectors $x_{k}$ are defined by $x_{k, i}=x_{k, i+3}$.

For growing $N\left(N_{\mathrm{p}}\right)$, the difference between the $x_{1}, j$ diminishes monotonically and approaches zero when $N\left(N_{\mathrm{p}}\right)$ tends to infinity. Figure A.1 summarizes the results for two particular choices of the light factors. We conclude that the spurious pattern caused by the regular spacing of the Doppler shifts is of practical importance for the reconstruction of small spectral intervals (single lines) only.

With observed spectra different from flat continua, the three $x_{k, j}$ would differ, as in (A.13), because of different line blocking in the sets of bins $x_{k, j+3 i}, j+3 i \leq N$. However, the basic result that the amplitude tends to zero in large spectral intervals remains valid.

\section{References}

Bagnuolo, W. G., Jr., \& Gies, D. R. 1991, ApJ, 376, 266

Bakis, V., Bakis, H., Eker, Z., \& Demircan, O. 2007, MNRAS, 382, 609

Fitzpatrick, E. L., Ribas, I., Guinan, E. F., DeWarf, L. E., \& Maloney, F. P. 2002 , ApJ, 564, 260

Frémat, Y., Lampens, P., \& Hensberge, H. 2005, MNRAS, 356, 545

Gies, D. R. 2004, in Spectroscopically and Spatially Resolving the Components of Close Binary Stars, ed. R. W. Hilditch, H. Hensberge, \& K. Pavlovski, ASP Conf. Ser., 318, 61 
González, J. F., \& Levato, H. 2006, A\&A, 448, 283

González, J. F., Hubrig, S., Nescavil, N., \& North, P. 2006, A\&A, 449, 327 Griffin, R. E. 2002, AJ, 123, 988

Hadrava, P. 1995, A\&AS, 114, 393

Hadrava, P. 1997, A\&AS, 122, 581

Hadrava, P. 2004a, in Spectroscopically and Spatially Resolving the Components of Close Binary Stars, ed. R. W. Hilditch, H. Hensberge, \& K. Pavlovski, ASP Conf. Ser., 318, 86

Hadrava, P. 2004b, Publ. Astron. Inst. ASCR 92, 15

Hadrava, P. 2006, A\&A, 448, 1149

Harries, T. J., Hilditch, R. W., \& Howarth, I. D. 2003, MNRAS, 339, 157

Hensberge, H., \& Pavlovski, K. 2007, in Binary Stars as Critical Tools and Tests in Contemporary Astrophysics, ed. W. I. Hartkopf, E. F. Guinan, \& P. Harmanec, IAU Symp., 240, 136

Hensberge, H., Pavlovski, K., \& Verschueren, W. 2000, A\&A, 358, 553

Hensberge, H., Vaz, L. P. R., Torres, K. B. V., \& Armond, T. 2007, in Multiple Stars across the HR Diagram, ESO Astrophysics Symposia, ed. Hubrig, PetrGotzens, \& Tokovinin, 47

Holmgren, D. E. 2004, in Spectroscopically and Spatially Resolving the Components of Close Binary Stars, ed. R. W. Hilditch, H. Hensberge, \& K. Pavlovski, ASP Conf. Ser., 318, 95
Holmgren, D., Hadrava, P., Harmanec, P., Koubsky, P., \& Kubat, J. 1997, A\&A, 322,565

Hynes, R. I., \& Maxted, P. F. L. 1998, A\&A, 331, 167

Ilijić, S. 2004, in Spectroscopically and Spatially Resolving the Components of Close Binary Stars, ed. R. W. Hilditch, H. Hensberge, \& K. Pavlovski, ASP Conf. Ser., 318, 107

Ilijić, S., Hensberge, H., \& Pavlovski, K. 2001, Fizika B, 10, 357

Lacy, C. H. S., Torres G., Claret, A., \& Vaz, L. P. R. 2005, AJ, 130, 2838

Press, W. H., Teukolsky, S. A., Vetterling, W. T., \& Flannery, B. P. 1992, Numerical Recipes, Chap. 15.4 (Cambridge University Press)

Pavlovski, K., \& Hensberge, H. 2005, A\&A, 439, 309

Simon, K. P., \& Sturm, E. 1994, A\&A, 281, 286

Thrall, R. M., \& Tornheim, L. 1966, Vector Spaces and Matrices 127 (New York: John Wiley \& Sons, Inc.), 127

Uytterhoeven, K., Harmanec, P., Telting, J. H., \& Aerts, C. 2005, A\&A, 440, 249

Vaz, L. P. R., Hensberge, H., Torres, K. B. V., et al. 2008, in preparation Zucker, S., \& Mazeh, T. 1994, ApJ, 420, 806

Zucker, S., Torres, G., \& Mazeh, T. 1995, ApJ, 452, 863 